\title{
Solution of a second order difference equation using the bilinear relations of Riemann
}

\author{
Stéphane R. Legault \\ Defence Research Establishment Ottawa, Ottawa, Ontario K1A 0Z4, Canada
}

Thomas B. A. Senior

Radiation Laboratory, Department of Electrical Engineering and Computer Science, The University of Michigan, Ann Arbor, Michigan 48109-2122

(Received 11 October 2000; accepted for publication 3 December 2001)

\begin{abstract}
A recently proposed technique to solve a class of second order functional difference equations arising in electromagnetic diffraction theory is further investigated by applying it to a case of intermediate complexity. The proposed approach is conceptually simple and relies on first obtaining well-defined branched solutions to a pair of associated first order difference equations. The construction of these branched expressions leads to an equation system whose solution requires relationships akin to Riemann's bilinear relations for differentials of the first and third kinds; their derivation necessitates the application of Cauchy's theorem on Riemann surfaces of, in this particular instance, genera one and three. Branch-free solutions of the second order difference equation are then obtained by taking appropriate linear combinations of the branched solutions of the first order equations. Analysis and computation demonstrate that the resulting expressions have the desired analytical properties and recover known solutions in the appropriate limit. (C) 2002 American Institute of Physics. [DOI: 10.1063/1.1445287]
\end{abstract}

\section{INTRODUCTION}

The Sommerfeld-Maliuzhinets technique ${ }^{1,2}$ remains today the most general approach to solve electromagnetic scattering problems involving wedge-shaped structures with faces characterized by impedance boundary conditions ${ }^{3}$ under plane wave illumination. Within its framework solutions are sought by expressing components of the total electric and magnetic fields in terms of unknown plane wave angular spectra, defined here in the complex $\alpha$ plane, which, besides being meromorphic, must satisfy a number of analyticity requirements. In particular, since poles of the spectral functions give rise to plane wave contributions, the spectra are required to be analyticsave for a pole necessary to reproduce the illuminating incident field - in a strip of the complex plane corresponding to the angular opening of the wedge. Additionally, besides the boundary conditions characterizing the surfaces of the wedge, obtaining a unique solution of the wave equation also requires knowledge of the behavior of the fields at the tip of the wedge and enforcement of the resulting edge condition ${ }^{4}$ specifies the asymptotic behavior ${ }^{2,3}$ of the spectral functions for large imaginary values of $\alpha$. The imposition of the boundary conditions on the spectral representation of the fields together with a theorem put forward by Maliuzhinets ${ }^{5}$ leads to a pair of first order difference equations for the spectra and their periods are related to the open angle of the wedge. The problem is thus reduced to obtaining spectra that satisfy both the difference equations as well as the analyticity requirements outlined above.

In the special case of normal incidence, where the illuminating plane wave is incident perpendicularly to the edge of the structure, the technique leads to uncoupled first order difference equations whose coefficients are rational trigonometric functions and solutions subject to the required constraints are readily obtained in terms of Maliuzhinets functions. ${ }^{1}$ At skew (nonnormal) incidence, the equation pair is generally coupled and solutions are obtainable for a few particular wedge/angle combinations for which uncoupled first order equations for linear combi- 
nations of the spectra can be found. Recent examples of this include the polarization independent wedge studied by Bernard ${ }^{6}$ and the right-angled anisotropic impedance wedge with one perfectly conducting face examined by Manara and Nepa. ${ }^{7}$ In general the equation pair cannot be uncoupled and we are faced with solving a second order functional difference equation whose coefficients are rational functions of trigonometric polynomials. Its solutions are linear combinations of the desired spectral functions, a consequence of the decoupling procedure, and must therefore satisfy analyticity requirements analogous to those of the spectral functions. A pair of associated first order difference equations can be obtained from the second order one, but these, as we shall see below, typically involve branched functions and Maliuzhinets's technique does not apply.

There have been few attempts published in the literature to solve second order difference equations due to the complicated nature of the problem. A successful example is provided by Gaudin $^{8}$ who considers the second order difference equation that arises in the study of the quantum mechanical problem of two electrons interacting with a localized magnetic moment. The particular equation studied is of a high order of complexity and the ensuing analysis is prohibitively complicated. In electromagnetic theory, the second order functional difference equation of form

$$
t(\alpha+3 \pi)-2\left\{1-2 \frac{\cos ^{2} \eta-\cos ^{2} \theta}{\cos ^{2} \alpha-\cos ^{2} \theta}\right\} t(\alpha+\pi)+t(\alpha-\pi)=0
$$

was recently solved by Senior and Legault. ${ }^{9}$ It is a generalization of the one considered by Demetrescu et al. ${ }^{10}$ in their study of the penetrable composite right-angled wedge consisting of abutted resistive and perfectly conducting semi-infinite half-planes. In this particular instance, the parameters $\eta$ and $\theta$ are both related to the resistivity of the wedge. As noted above, the function $t(\alpha)$ represents a combination of the unknown spectral functions and it therefore satisfies requirements related to those imposed on the spectral functions. Accordingly, the solutions $t(\alpha)$ obtained in Ref. 9 are ( $i$ ) meromorphic, ( $i i)$ free of poles and zeros in the strip of analyticity $\mathcal{S}_{2 \pi}$ $=\{\alpha:|\operatorname{Re} \alpha| \leqslant \pi\}$ (the inclusion of zeros here is a consequence of reciprocal symmetry between solution pairs in certain limits), and, in accordance with the edge condition, (iii) $\mathcal{O}(1)$ as $|\operatorname{Im} \alpha| \rightarrow \infty$. Two linearly independent solutions satisfying the above analyticity requirements were constructed by successively eliminating the undesired singularities in the strip $\mathcal{S}_{2 \pi}$. The conceptual simplicity of the technique hinges on recognizing that expressions recovered during the course of the analysis are of the same nature as those occurring in Riemann's bilinear relations for differentials of the first and third kinds. ${ }^{11,12}$ In contrast, the solution based on a Fourier transform approach proposed in Ref. 10 fails to satisfy requirement $(i)$ above since it is free of branch points only in the strip of analyticity $\mathcal{S}_{2 \pi}$ as opposed to the entire $\alpha$ plane.

Equation (1) may be qualified as being of moderate complexity due to the relatively low number of singularities (poles and branch points) which must be eliminated to successfully complete the analysis. In comparison, geometries of contemporary interest such as the right-angled wedge characterized by isotropic impedance boundary conditions on both faces or the anisotropic impedance half-plane (see Ref. 13 for an approximate solution) lead to substantially more complicated equations. To gain insight into the applicability of the technique in such cases and also provide some details on the procedure, as opposed to focusing on a particular physical problem, we examine here a case of intermediate complexity by considering an equation of the same form as (1) but with the period doubled to $4 \pi$, viz.

$$
t(\alpha+5 \pi)-2\left\{1-2 \frac{\cos ^{2} \eta-\cos ^{2} \theta}{\cos ^{2} \alpha-\cos ^{2} \theta}\right\} t(\alpha+\pi)+t(\alpha-3 \pi)=0,
$$

and, consistent with the requirement for (1), a solution is sought which is (i) meromorphic, (ii) free of poles and zeros in the strip of analyticity $\mathcal{S}_{4 \pi}=\{\alpha:|\operatorname{Re} \alpha| \leqslant 2 \pi\}$, and (iii) $\mathcal{O}(1)$ as $|\operatorname{Im} \alpha|$ $\rightarrow \infty$. The increase in complexity arises from the doubling of the strip of analyticity as this effectively doubles the number of singularities, both poles and branch points, that must be con- 
sidered in the course of the analysis. Consequently, whereas the solution for (1) required analysis on a Riemann surface corresponding to a torus (handlebody of genus one), in the case of (2) it will be seen that we are required to work on Riemann surfaces whose corresponding handlebodies are of genera one and three.

In what follows, Sec. II gives the solution procedure for the branched first order equations associated with (2) and provides the derivation of a system of four equations in four unknowns to be satisfied in order for their solutions to be well-defined. The unknowns consist of two multiplicative constants associated with elliptic integrals of the first kind with periods $2 \pi$ and $4 \pi$, and the location of the logarithmic singularities associated with two elliptic integrals of the third kind also of periods $2 \pi$ and $4 \pi$. Section III shows how to solve for the quantities associated with the $2 \pi$ periodic elliptic integrals. The analysis, which is carried out on a torus, is of the same nature as that required in Ref. 9 but it is examined here in greater detail. Section IV gives the solution for the quantities associated with the elliptic integrals of period $4 \pi$ and it is now required to carry out the analysis on a Riemann surface which is the topological equivalent of a handlebody of genus three. The branched solutions to the first order equations are used in Sec. V to construct branchfree solutions to the second order equation. A fully analytic solution that satisfies all of the prescribed requirements is provided. The only shortcoming of the solution is that it vanishes in a certain limit and, in an effort to address this shortcoming, an alternative approach that relies on numerically locating zeros is also examined.

\section{FIRST ORDER EQUATIONS AND SOLUTIONS}

Since there is no available technique to directly attack the type of second order difference equation with which we are concerned, it must first be recast as an associated pair of more easily handled first order difference equations. Unfortunately the latter generally involve branch points; the price paid for this reduction in order is that the established solution technique for first order equations by Maliuzhinets ${ }^{1}$ fails to apply. However, solutions to the first order difference equations can, in principle at least, be obtained by applying a logarithmic derivative and this is the approach taken here. This yields a solution expressed in terms of an initially ill-defined path integral and multiplicative terms of period $4 \pi$, corresponding to the period of the difference equation, must be added to rectify this. This ultimately leads to the derivation of a system of four equations in four unknowns which can be partially decoupled into two systems in two unknowns, one involving $2 \pi$ periodic quantities and the other $4 \pi$ periodic ones.

\section{A. Reduction to first order equations}

The second order functional difference equation (2) can be rewritten in terms of first order difference equations quite straightforwardly by exploiting the periodicity of the functional coefficient. To see this consider the second order difference equation

$$
t(\alpha+5 \pi)+p(\alpha) t(\alpha+\pi)+\frac{1}{p(\alpha)}\{t(\alpha+\pi)+p(\alpha) t(\alpha-3 \pi)\}=0
$$

whose solutions $t(\alpha)$, provided $p(\alpha)$ is $4 \pi$ periodic, must also explicitly satisfy first order difference equations. Enforcing equality between (3) and (2) then yields the equation pair

$$
\begin{aligned}
& \frac{t(\alpha+2 \pi)}{t(\alpha-2 \pi)}=g(\alpha,+u(\alpha))=\frac{u(\alpha)-u(\theta)}{u(\alpha)+u(\theta)}, \\
& \frac{t(\alpha+2 \pi)}{t(\alpha-2 \pi)}=g(\alpha,-u(\alpha))=\frac{u(\alpha)+u(\theta)}{u(\alpha)-u(\theta)},
\end{aligned}
$$

where

$$
u(\alpha)=\sqrt{\cos ^{2} \alpha-\cos ^{2} \eta}
$$




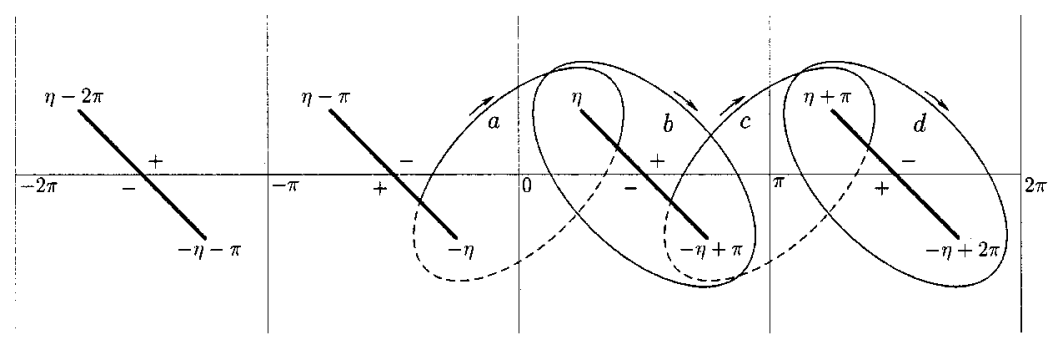

FIG. 1. The strip of analyticity $\mathcal{S}_{4 \pi}=\{\alpha:|\operatorname{Re} \alpha| \leqslant 2 \pi\}$. The thick lines indicate the branch cuts of $u(\alpha)$, the positive and negative signs indicate relative changes in sign of $u(\alpha)$ across the different cuts. The clockwise cycles $a, b, c$ and $d$ used to define the cyclic periods are as indicated. Note that the cycles $a$ and $c$ cross from the upper Riemann sheet (solid line) to the lower Riemann sheet (dashed line) whereas $b$ and $d$ are confined to the upper sheet.

Alternatively, one could also proceed by factoring the associated second order difference operator in (2) into a pair of first order difference operators and recover the same result as above. The first order equations obtained are generally branched owing to the presence of the root $u(\alpha)$ which has branch points at $\alpha= \pm \eta, \pm(\eta-\pi), \pm(\eta+\pi), \pm(-\eta+2 \pi)$ in the strip of analyticity $\mathcal{S}_{4 \pi}$ and is made well defined in the complex $\alpha$ plane by introducing the cuts shown in Fig. 1. These are such that $u(\alpha)$ has the same symmetry as $\cos \alpha$ so that

$$
u(\alpha)=u(-\alpha)=-u(\alpha \pm \pi) .
$$

Note that the function $t(\alpha)$ is used here to generally denote solutions of the second order equation and each of the first order equations above identifies one such solution. If $t(\alpha)=w(\alpha, u(\alpha))$ is a solution of (4), then $t(\alpha)=w(\alpha,-u(\alpha))$ is a solution of (5), and this follows from the symmetry of the right-hand sides of (4) and (5) with respect to the sign (the branch) of $u(\alpha)$. It is therefore sufficient to consider $w(\alpha, u)$-writing $w(\alpha, u)$ instead of $w(\alpha, u(\alpha))$ for convenience-and, since $g(-\alpha, u)=g(\alpha, u)$, we can construct $w(\alpha, u)$ such that

$$
w(-\alpha, u)=\frac{1}{w(\alpha, u)}=w(\alpha,-u)
$$

In terms of the solutions of the first order difference equations, solutions to (2) are

$$
t(\alpha)=C_{1}(\alpha) w(\alpha, u)+C_{2}(\alpha) w(\alpha,-u),
$$

where $C_{1,2}(\alpha)$ are $4 \pi$ periodic functions. This generally conflicts with the requirement for a branch-free solution but there are particular cases of (9) that overcome this difficulty and the simplest two such linearly independent expressions free of branch points are

$$
t(\alpha)=w(\alpha, u)+w(\alpha,-u), \quad \text { and } \quad t(\alpha)=\frac{1}{u(\alpha)}\{w(\alpha, u)-w(\alpha,-u)\} .
$$

While the branch-free property of these two symmetric forms can be ascertained rigorously by means of Taylor expansions in the neighborhood of branch points of $u(\alpha)$, it can also be appreciated from the fact that both are invariant under a change of the branch of $u(\alpha)$. This crucial feature makes the constructs (10) the fundamental building blocks from which branch-free solutions to the second order difference equation can be assembled once branched solutions of the associated first order equations have been obtained. As we shall soon see, the presence of branch points makes this task quite challenging and the brunt of the subsequent analysis focuses on deriving solutions to the first order equations. 


\section{B. Special cases of interest}

It is worthwhile to first consider the special cases $\eta=0$ and $\eta=\pi / 2$ as the branch points then vanish and known solutions, which are useful when characterizing the behavior of the general solution obtained below, can be obtained in terms of Maliuzhinets functions. The first one when $\eta=\pi / 2$ proves to be especially interesting since the branch cuts of $u(\alpha)$, as illustrated in Fig. 1 and chosen so that (7) holds, vanish as $\eta \rightarrow \pi / 2$. In this instance (4) becomes

$$
\frac{w(\alpha+2 \pi, u)}{w(\alpha-2 \pi, u)}=\frac{\cos \alpha-\cos \theta}{\cos \alpha+\cos \theta},
$$

and, despite the fact that the right-hand side is now meromorphic, the dependence of $w(\alpha, u)$ on $u(\alpha)$ is maintained to distinguish it from $w(\alpha,-u)$, the solution to Eq. (5) in the same limit. A solution free of poles and zeros in $\mathcal{S}_{4 \pi}$ and $\mathcal{O}(1)$ as $|\operatorname{Im} \alpha| \rightarrow \infty$ follows directly from Maliuzhinets. ${ }^{1}$ It may be written as

$$
\begin{aligned}
& w(\alpha, u)=\Psi_{1}(\alpha) \\
& =\frac{\psi_{\pi}(\alpha+\pi / 2-\theta)}{\psi_{\pi}(\alpha-\pi / 2+\theta)}
\end{aligned}
$$

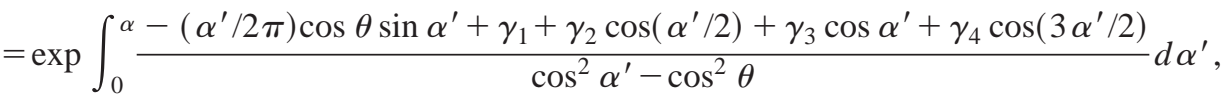

where

$$
\begin{gathered}
\gamma_{1}=-\frac{1}{4} \sin \theta \cos \theta, \\
\gamma_{2}=\frac{1}{4}\left(-\cos \frac{\theta}{2}+\sin \frac{\theta}{2}\right)+\frac{1}{2} \cos \frac{\theta}{2}\left(\cos \frac{\theta}{2}+\sin \frac{\theta}{2}\right), \\
\gamma_{3}=\left(\frac{\theta}{2 \pi}-\frac{1}{4}\right) \sin \theta, \\
\gamma_{4}=\frac{1}{4}\left(-\cos \frac{\theta}{2}+\sin \frac{\theta}{2}\right),
\end{gathered}
$$

and $\psi_{\pi}(\alpha)$ is the Maliuzhinets function. ${ }^{1}$ Since $\Psi_{1}(-\alpha)=1 / \Psi_{1}(\alpha)$, consistent with (8), two linearly independent solutions of (2) are

$$
t_{1}(\alpha)=\Psi_{1}(\alpha), \quad t_{2}(\alpha)=\Psi_{1}(-\alpha),
$$

and these are both $\mathcal{O}(1)$ as $|\operatorname{Im} \alpha| \rightarrow \infty$. The other case of interest where the branch points also vanish is $\eta=0$ and in that instance two linearly independent solutions, also $\mathcal{O}(1)$ as $|\operatorname{Im} \alpha| \rightarrow \infty$, are provided by

$$
t_{1}(\alpha)=\Psi_{2}(\alpha)=\cos \frac{\alpha}{4} \frac{\psi_{\pi}^{2}(\pi / 2-\theta)}{\psi_{\pi}(\alpha+\pi / 2-\theta) \psi_{\pi}(\alpha-\pi / 2+\theta)}, \quad t_{2}(\alpha)=\frac{1}{t_{1}(\alpha)},
$$

and we must now contend with poles or zeros at $\alpha= \pm 2 \pi$. 


\section{General case}

We now proceed to solve (4) in the general case where $\eta \neq 0, \pi / 2$ and Maliuzhinets's technique does not apply. The solutions sought should preferably reduce to one or both of the known limiting functions when $\eta$ goes to the appropriate limit and, consistent with the limiting cases, they must also be $\mathcal{O}(1)$ as $|\operatorname{Im} \alpha| \rightarrow \infty$ for all $\eta$. As we shall see, while the solution $\Psi_{1}(\alpha)$ [and $\left.1 / \Psi_{1}(\alpha)\right]$ are easily recovered as $\eta \rightarrow \pi / 2$, the recovery of $\Psi_{2}(\alpha)$ [and $\left.1 / \Psi_{2}(\alpha)\right]$ as $\eta \rightarrow 0$ proves to be much more difficult. Taking the logarithmic derivative of (4), we obtain

$$
\frac{d}{d \alpha} \ln w(\alpha+2 \pi, u)-\frac{d}{d \alpha} \ln w(\alpha-2 \pi, u)=\frac{d}{d \alpha} \ln g(\alpha, u)=-\frac{u(\theta)}{u(\alpha)} \frac{2 \sin \alpha \cos \alpha}{\cos ^{2} \alpha-\cos ^{2} \theta},
$$

and if $v_{0}(\alpha, u)=d / d \alpha \ln w(\alpha, u)$, then

$$
v_{0}(\alpha+2 \pi, u)-v_{0}(\alpha-2 \pi, u)=-\frac{u(\theta)}{u(\alpha)} \frac{2 \sin \alpha \cos \alpha}{\cos ^{2} \alpha-\cos ^{2} \theta}
$$

and a solution to (4) can tentatively be written as

$$
w(\alpha, u)=\exp \int_{\alpha_{0}}^{\alpha} v_{0}\left(\alpha^{\prime}, u\right) d \alpha^{\prime},
$$

with

$$
v_{0}(\alpha, u)=-\frac{\alpha}{2 \pi} \frac{u(\theta)}{u(\alpha)} \frac{\sin \alpha \cos \alpha}{\cos ^{2} \alpha-\cos ^{2} \theta} .
$$

The form proposed in (15) is, however, ill-defined owing to the presence of the polar and the cyclic periods (we borrow here the terminology used in Ref. 11 when characterizing differentials of the third kind) due to, respectively, the poles and the branch points of $v_{0}(\alpha, u)$. In order to obtain a single-valued integral expression, we must consider instead

$$
w(\alpha, u)=\exp \int_{\alpha_{0}}^{\alpha}\left\{v_{0}\left(\alpha^{\prime}, u\right)+v_{\Sigma}\left(\alpha^{\prime}, u\right)\right\} d \alpha^{\prime},
$$

where the added term $v_{\Sigma}(\alpha, u)$ represents a sum of $4 \pi$ periodic terms, of even parity like $v_{0}(\alpha, u)$, specifically selected to remove the offending periods. Not all classes of $4 \pi$ periodic functions are acceptable: it turns out that $v_{\Sigma}(\alpha, u)$ must fulfill certain order requirements in order for $\exp \int v_{\Sigma}(\alpha) d \alpha$ to be a $4 \pi$ periodic function and a simple analysis shows that, for the case at hand, it is sufficient to consider expressions such that $v_{\Sigma}(\alpha, u) \rightarrow 0$ as $|\operatorname{Im} \alpha| \rightarrow \infty$. It will be shown below that $v_{\Sigma}(\alpha, u)$ will consist of five terms: $v_{1}(\alpha, u)$ to eliminate the polar periods and $v_{2 \pi}^{1}(\alpha, u), v_{2 \pi}^{3}(\alpha, u), v_{4 \pi}^{1}(\alpha, u), v_{4 \pi}^{3}(\alpha, u)$ to eliminate the cyclic periods.

It is worthwhile to discuss the nature of the lower limit $\alpha_{0}$ at this juncture as its selection may appear at first glance to be somewhat arbitrary. This is not the case as consideration of the symmetric forms in (10) together with the requirement for continuity reveals that the lower limit $\alpha_{0}$ must be a branch point in $\mathcal{S}_{4 \pi}$ so that $\alpha_{0} \in\{ \pm \eta, \pm(\eta-\pi), \pm(\eta+\pi), \pm(2 \pi-\eta)\}$. Furthermore, once $v_{\Sigma}(\alpha, u)$ has been properly defined, the solution is independent of the choice of the particular branch point and this will become more obvious when we consider the elimination of the cyclic periods. We first examine the elimination of the polar periods.

\section{Elimination of polar periods}

The presence of poles will generally make a path integral such as the one in (16) multivalued. A pole of residue Res will give rise to a polar period equal to $2 \pi i$ Res and, depending on the orientation of the integration path and its winding number around the pole, the contribution to the integral will be $2 \pi i$ ZRes. In the case of (15), polar periods arise at the poles of $v_{0}(\alpha, u)$ at $\alpha$ 
$= \pm \theta, \pm(\theta-\pi), \pm(\theta+\pi), \pm(2 \pi-\theta)$. Elimination of these poles serves two purposes as it not only eliminates their associated polar periods but goes toward fulfilling the requirement for a solution that is pole-free in $\mathcal{S}_{4 \pi}$. We eliminate them by introducing the $4 \pi$ periodic

$$
v_{1}(\alpha, u)=\frac{u(\theta)}{u(\alpha)} \frac{\cos (\alpha)}{\cos (\theta)} \frac{\gamma_{1}+\gamma_{2} \cos (\alpha / 2)+\gamma_{3} \cos \alpha+\gamma_{4} \cos (3 \alpha / 2)}{\cos ^{2} \alpha-\cos ^{2} \theta},
$$

which is even, vanishes as $|\operatorname{Im} \alpha| \rightarrow \infty$, and has poles coinciding with those of $v_{0}(\alpha, u)$. The constants $\gamma_{n}$ are chosen to eliminate the residues and straightforward algebra yields the coefficients in (12). It then follows from (11) that

$$
v_{0}(\alpha, u)+v_{1}(\alpha, u)=\frac{u(\theta)}{u(\alpha)} \frac{\cos \alpha}{\cos \theta} \frac{d}{d \alpha} \ln \Psi_{1}(\alpha),
$$

which correctly reduces to $d / d \alpha \ln \Psi_{1}(\alpha)$ when $\eta=\pi / 2$. We therefore recognize that, in the simpler case where the right-hand side of (14) is meromorphic so that (15) is free of cyclic periods, the known solutions expressed in terms of Maliuzhinets can be recovered by following the above procedure of pole elimination. We also note in passing that poles with integer residue $\mathbb{Z}$ do not compromise path independence. Indeed, their capture leads to an additive $2 \pi i Z$ contribution in the exponent of (16) which has no effect on the final value of $w(\alpha, u)$.

\section{Elimination of cyclic periods}

In a fashion similar to polar periods, a cyclic period arises from the nonzero contribution incurred when integrating along a loop encircling a branch cut in $\mathcal{S}_{4 \pi}$, thereby making the path integral multivalued. For example, such a period is obtained when integrating $v_{0}+v_{1}$ along the cycle $b$, shown in Fig. 1, which encircles the branch cut joining the branch points $\eta$ and $-\eta$ $+\pi$. As in the case of the polar periods, it is strictly speaking not required for the cyclic periods to vanish identically to avoid jeopardizing single-valuedness since periods equal to $2 \pi i Z$ do not change the value of (16). However, as $\eta \rightarrow \pi / 2$ the branch points of $1 / u(\alpha)$ in $v_{0,1}$ coalesce into poles at $\pm \pi / 2$ and $\pm 3 \pi / 2$ and their associated cyclic periods then become polar periods. Consequently, the cyclic periods associated with the cycles $b$ and $d$ in Fig. 1 must be annulled, as opposed to setting them equal to some nonzero integer multiple of $2 \pi i$, to eliminate poles that would otherwise arise as $\eta \rightarrow \pi / 2$. We observe that this requirement is equivalent to annulling the integral of $v_{0}+v_{\Sigma}$ along the cuts between $\eta$ and $\pi-\eta$ as well as $\pi+\eta$ and $2 \pi-\eta$. There is also a similar requirement, which is not obvious when solely considering integration on either of the Riemann sheets, on the cyclic periods associated with the cycles $a$ and $c$ which loop from one Riemann sheet to the other. Its necessity is revealed by examining either of the symmetric forms in (10) together with the requirement for continuity. In short, the above implies, taking advantage of the even parity, the need to annul the cyclic periods of $v_{0}(\alpha, u)+v_{\Sigma}(\alpha, u)$ on the clockwise cycles $a, b, c$ and $d$ shown in Fig. 1. Alternatively, this can be thought of as requiring that all branch point to branch point integrals vanish in $\mathcal{S}_{4 \pi}$ and, under this condition, the lower limit $\alpha_{0}$ can be arbitrarily chosen among any of the branch points located within the strip. This also ensures that the resulting expressions will remain free of poles despite coalescing branch points in the limits $\eta \rightarrow 0$ and $\eta \rightarrow \pi / 2$.

Four degrees of freedom are required to annul the cyclic periods of $v_{0}+v_{1}$ on the cycles $a$, $b, c$ and $d$. We introduce the following four even $4 \pi$ periodic terms which, like $v_{0}(\alpha, u)$ and $v_{1}(\alpha, u)$, vanish as $|\operatorname{Im} \alpha| \rightarrow \infty$ :

$$
v_{2 \pi}^{1}(\alpha, u)=\frac{1}{u(\alpha)}, \quad v_{2 \pi}^{3}(\alpha, u)=\frac{u\left(\zeta_{2 \pi}\right)}{u(\alpha)} \frac{\cos \alpha}{\cos \zeta_{2 \pi}} \frac{\sin \zeta_{2 \pi}}{\cos \alpha-\cos \zeta_{2 \pi}},
$$




$$
v_{4 \pi}^{1}(\alpha, u)=\frac{\cos (\alpha / 2)}{u(\alpha)}, \quad v_{4 \pi}^{3}(\alpha, u)=\frac{u\left(\zeta_{4 \pi}\right)}{u(\alpha)} \frac{\cos \alpha}{\cos \zeta_{4 \pi}} \frac{\frac{1}{2} \sin \left(\zeta_{4 \pi} / 2\right)}{\cos (\alpha / 2)-\cos \left(\zeta_{4 \pi} / 2\right)}
$$

These all give rise to elliptic integrals with the first pair being $2 \pi$ periodic and the second $4 \pi$ periodic. The subscript identifies the periodicity of the term while the superscript identifies the type of elliptic integral to which it gives rise. Hence $v_{2 \pi}^{1}(\alpha, u)$ is $2 \pi$ periodic and gives rise to an elliptic integral of the first kind while $v_{2 \pi}^{3}(\alpha, u)$, also $2 \pi$ periodic, gives rise to an elliptic integral of the third kind with logarithmic singularities within $\mathcal{S}_{4 \pi}$ at $\pm \zeta_{2 \pi}, \pm\left(\zeta_{2 \pi}+2 \pi\right)$. Likewise, $v_{4 \pi}^{3}(\alpha, u)$ is $4 \pi$ periodic and gives rise to an elliptic integral of the third kind with logarithmic singularities at $\pm \zeta_{4 \pi}$. The use of expressions associated with integrals of the third kind (with poles having nonvanishing residues) results from the impossibility of introducing the required number of degrees of freedom without violating the order requirement. It must be emphasized that the poles of both $v_{2 \pi}^{3}(\alpha, u)$ and $v_{4 \pi}^{3}(\alpha, u)$ have residues \pm 1 and their polar periods therefore do not disrupt the single-valuedness of the path integral. Their elimination from the strip of analyticity is the objective of the last step in the construction of the solution and this is carried out in Sec. V. For future reference we define the cyclic periods

$$
\begin{aligned}
& A_{2 \pi, 4 \pi}^{1,3}=\int_{a} v_{2 \pi, 4 \pi}^{1,3}(\alpha, u) d \alpha, \quad B_{2 \pi, 4 \pi}^{1,3}=\int_{b} v_{2 \pi, 4 \pi}^{1,3}(\alpha, u) d \alpha \\
& C_{2 \pi, 4 \pi}^{1,3}=\int_{c} v_{2 \pi, 4 \pi}^{1,3}(\alpha, u) d \alpha, \quad D_{2 \pi, 4 \pi}^{1,3}=\int_{d} v_{2 \pi, 4 \pi}^{1,3}(\alpha, u) d \alpha,
\end{aligned}
$$

and use similar definitions for $v_{0,1}(\alpha, u)$ [i.e., $A_{0}=\int_{a} v_{0}(\alpha, u) d \alpha$ ]. Inspection of (17) and (18) reveals that the periods associated with the integrals of the third kind are functions of the poles $\zeta_{2 \pi}$ and $\zeta_{4 \pi}$, providing two of the four degrees of freedoms required to annul the periods of $v_{0}(\alpha, u)+v_{\Sigma}(\alpha, u)$. In contrast, the periods associated with the integrals of the first kind are constant and two multiplicative constants, $\kappa_{2 \pi}$ and $\kappa_{4 \pi}$, must be introduced to produce the two additional degrees of freedom. The solution to (4) then takes the form

$$
\begin{aligned}
w(\alpha, u)= & \exp \int_{\alpha_{0}}^{\alpha}\left\{v_{0}\left(\alpha^{\prime}, u\right)+v_{1}\left(\alpha^{\prime}, u\right)+\kappa_{2 \pi} v_{2 \pi}^{1}\left(\alpha^{\prime}, u\right)+\sigma_{2 \pi} v_{2 \pi}^{3}\left(\alpha^{\prime}, u\right)\right. \\
& \left.+\kappa_{4 \pi} v_{4 \pi}^{1}\left(\alpha^{\prime}, u\right)+\sigma_{4 \pi} v_{4 \pi}^{3}\left(\alpha^{\prime}, u\right)\right\} d \alpha^{\prime}
\end{aligned}
$$

where the four unknowns to be determined are $\kappa_{2 \pi}, \zeta_{2 \pi}$ and $\kappa_{4 \pi}, \zeta_{4 \pi}$. The quantities $\sigma_{2 \pi}$ $= \pm 1$ and $\sigma_{4 \pi}= \pm 1$ have been introduced to avoid loss of generality in the definition of the terms associated with the integrals of the third kind. They account for the eventuality where the sign of the logarithmic residues of $v_{2 \pi}^{3}(\alpha, u)$ or $v_{4 \pi}^{3}(\alpha, u)$ must be changed, thereby swapping poles and zeros of $w(\alpha, u)$ between the two Riemann sheets. Their proper definition will be determined in the course of the analysis and, to reduce clutter, they will be omitted in what follows pending their reintroduction when appropriate.

An equation system consisting of four equations in the four unknowns is obtained by enforcing vanishing cyclic periods on the cycles $a, b, c$ and $d$. Doing so for the cycle $d$, for example, leads to

$$
\int_{\eta+\pi^{-}}^{-\eta+2 \pi^{-}}\left(v_{0}+v_{1}+\kappa_{2 \pi} v_{2 \pi}^{1}+v_{2 \pi}^{3}+\kappa_{4 \pi} v_{4 \pi}^{1}+v_{4 \pi}^{3}\right) d \alpha=0
$$

with the superscript negative sign in the limits indicating the corresponding side of the branch cut (see Fig. 1) along which to integrate. Upon use of (19) this becomes

$$
D_{0}+D_{1}+\kappa_{2 \pi} D_{2 \pi}^{1}+D_{2 \pi}^{3}+\kappa_{4 \pi} D_{4 \pi}^{1}+D_{4 \pi}^{3}=0
$$


which is further simplified by exploiting the symmetries $D_{2 \pi}^{1}=-B_{2 \pi}^{1}, D_{2 \pi}^{3}=-B_{2 \pi}^{3}$ and $D_{4 \pi}^{1}$ $=B_{4 \pi}^{1}$. Introducing the notation $D_{0+1}=D_{0}+D_{1}$, we finally obtain

$$
D_{0+1}-\kappa_{2 \pi} B_{2 \pi}^{1}-B_{2 \pi}^{3}+\kappa_{4 \pi} B_{4 \pi}^{1}+D_{4 \pi}^{3}=0,
$$

a relationship equivalent to (21). Repeating the same process for the $a, b$ and $c$ cycles yields

$$
\begin{gathered}
A_{0+1}+\kappa_{2 \pi} A_{2 \pi}^{1}+A_{2 \pi}^{3}+\kappa_{4 \pi} A_{4 \pi}^{1}+A_{4 \pi}^{3}=0, \\
B_{0+1}+\kappa_{2 \pi} B_{2 \pi}^{1}+B_{2 \pi}^{3}+\kappa_{4 \pi} B_{4 \pi}^{1}+B_{4 \pi}^{3}=0, \\
C_{0+1}-\kappa_{2 \pi} A_{2 \pi}^{1}-A_{2 \pi}^{3}+C_{4 \pi}^{3}=0, \\
D_{0+1}-\kappa_{2 \pi} B_{2 \pi}^{1}-B_{2 \pi}^{3}+\kappa_{4 \pi} B_{4 \pi}^{1}+D_{4 \pi}^{3}=0,
\end{gathered}
$$

with the explicit unknowns $\kappa_{2 \pi}$ and $\kappa_{4 \pi}$ and the unknowns $\zeta_{2 \pi}$ and $\zeta_{4 \pi}$ implied by the presence of cyclic periods associated with the integrals of the third kind. Note that $C_{4 \pi}^{1}$ vanishes since $v_{4 \pi}^{1}(\alpha, u)$, which is odd symmetric with respect to $\pi$ [see (18)], does not contribute when integrated on cycle $c$ (see Fig. 1). This seemingly intractable system can be fully solved analytically. The quantities associated with the $4 \pi$ periodic elliptic integrals can be decoupled by adding Eq. (22a) to $(22 \mathrm{c})$ and $(22 \mathrm{~b})$ to $(22 \mathrm{~d})$ to obtain

$$
\begin{gathered}
\kappa_{4 \pi} A_{4 \pi}^{1}+A_{4 \pi}^{3}+C_{4 \pi}^{3}=-\left(A_{0+1}+C_{0+1}\right), \\
2 \kappa_{4 \pi} B_{4 \pi}^{1}+B_{4 \pi}^{3}+D_{4 \pi}^{3}=-\left(B_{0+1}+D_{0+1}\right),
\end{gathered}
$$

and the elimination of $\kappa_{4 \pi}$ produces

$$
A_{4 \pi}^{1}\left(B_{4 \pi}^{3}+D_{4 \pi}^{3}\right)-2 B_{4 \pi}^{1}\left(A_{4 \pi}^{3}+C_{4 \pi}^{3}\right)=-A_{4 \pi}^{1}\left(B_{0+1}+D_{0+1}\right)+2 B_{4 \pi}^{1}\left(A_{0+1}+C_{0+1}\right)
$$

in which the only (implicit) unknown $\zeta_{4 \pi}$ determines the periods $A_{4 \pi}^{3}, B_{4 \pi}^{3}, C_{4 \pi}^{3}$ and $D_{4 \pi}^{3}$. Despite appearances, the above equation can be inverted to obtain $\zeta_{4 \pi}$ and the technique for doing so is described in Sec. IV. Once $\zeta_{4 \pi}$ has been obtained, the value of $\kappa_{4 \pi}$ immediately follows either from Eq. (23a) or (23b). One can then proceed to solve for $\zeta_{2 \pi}$ by subtracting Eq. (22c) from (22a) and (22d) from (22b) to obtain, respectively,

$$
\begin{gathered}
2 \kappa_{2 \pi} A_{2 \pi}^{1}+2 A_{2 \pi}^{3}=-\kappa_{4 \pi} A_{4 \pi}^{1}-A_{4 \pi}^{3}+C_{4 \pi}^{3}-A_{0+1}+C_{0+1}, \\
2 \kappa_{2 \pi} B_{2 \pi}^{1}+2 B_{2 \pi}^{3}=-B_{4 \pi}^{3}+D_{4 \pi}^{3}-B_{0+1}+D_{0+1},
\end{gathered}
$$

and the elimination of $\kappa_{2 \pi}$ gives

$$
\begin{aligned}
A_{2 \pi}^{1} B_{2 \pi}^{3}-B_{2 \pi}^{1} A_{2 \pi}^{3}= & \frac{1}{2}\left\{A_{2 \pi}^{1}\left(-B_{4 \pi}^{3}+D_{4 \pi}^{3}-B_{0+1}+D_{0+1}\right)\right. \\
& \left.-B_{2 \pi}^{1}\left(-\kappa_{4 \pi} A_{4 \pi}^{1}-A_{4 \pi}^{3}+C_{4 \pi}^{3}-A_{0+1}+C_{0+1}\right)\right\},
\end{aligned}
$$

where the only unknown is now $\zeta_{2 \pi}$, the value of which determines the periods $A_{2 \pi}^{3}$ and $B_{2 \pi}^{3}$. This equation is of the same form as the one obtained in Ref. 9 and the solution follows the same approach. To set the stage for the comparatively more complicated inversion required for $\zeta_{4 \pi}$, we first reexamine the analysis required for $\zeta_{2 \pi}$ in more detail.

\section{DETERMINATION OF $\zeta_{2 \pi}$ AND $\kappa_{2 \pi}$}

The key to inverting for $\zeta_{2 \pi}$ in (26) lies in the application of Cauchy's theorem on the Riemann surface delimited by the contour $\mathcal{C}_{1}$ shown in Fig. 2 . Indeed, by judiciously choosing the integrand it is possible to obtain an alternative expression for the left-hand side of (26) in which 


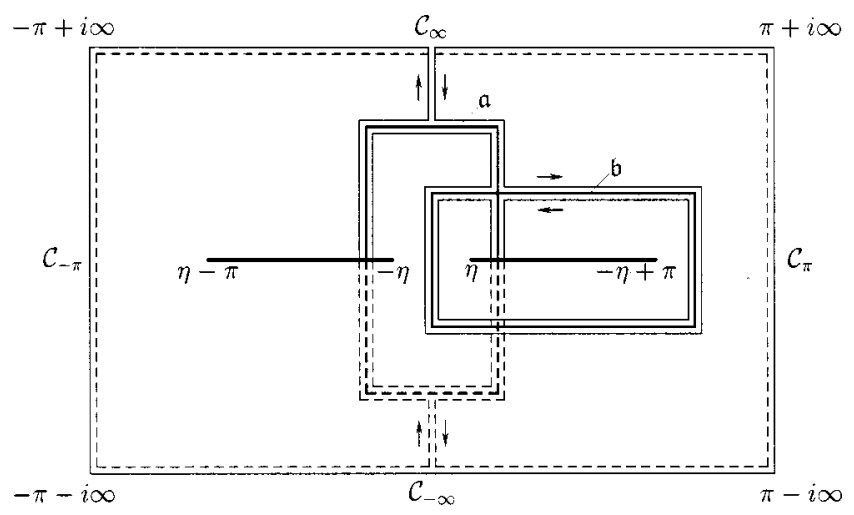

FIG. 2. The contour $\mathcal{C}_{1}=\mathcal{C}_{\mathfrak{a} \cup \mathfrak{b}} \cup \mathcal{C}_{ \pm \pi} \cup \mathcal{C}_{ \pm \infty}$ on the upper (solid line) and lower (dashed line) sheets of the Riemann surface. The thicker inner lines are the dissections $\mathfrak{a}$ and $\mathfrak{b}$ introduced to make the Riemann surface simply connected. The path $\mathcal{C}_{\mathfrak{a} \cup \mathfrak{b}}$ denotes the portion of the contour enclosing the dissecting cycles $\mathfrak{a}$ and $\mathfrak{b}$.

the unknown $\zeta_{2 \pi}$ appears more explicitly as the argument of an elliptic integral of the first kind, paving the way for its inversion by means of the Jacobian elliptic sine function. This follows from recognizing that the relationship between cyclic periods on the left-hand side of (26) is of the same form as the expressions found in Riemann's bilinear relationships for differentials of the first and third kinds: $;^{11,12}$ these equate expressions involving cyclic periods such as the one on the left-hand side of (26) to sums of residues. To achieve this, we seek to evaluate

$$
\int_{\mathcal{C}_{1}} V_{2 \pi}^{1}(\alpha, u) d V_{2 \pi}^{3}(\alpha, u)=2 \pi i \sum \operatorname{Res},
$$

where the elliptic integral $V_{2 \pi}^{1}(\alpha, u)$ of the first kind and $V_{2 \pi}^{3}(\alpha, u)$ of the third kind are defined as

$$
V_{2 \pi}^{n}(\alpha, u)=\int_{(\eta, 0)}^{(\alpha, u)} v_{2 \pi}^{n}\left(\alpha^{\prime}, u\right) d \alpha^{\prime}, \quad n \in\{1,3\}
$$

The path of integration $\mathcal{C}_{1}$, shown in Fig. 2, delimits a strip of width $2 \pi$ centered at the origin of both Riemann sheets and encloses the dissections and branch cuts contained therein. For $2 \pi$ periodic functions the enclosed surface is topologically equivalent to a torus (a handlebody of genus 1) as shown in Fig. 3. The canonical dissections $\mathfrak{a}$ and $\mathfrak{b}$ are introduced to make the surface

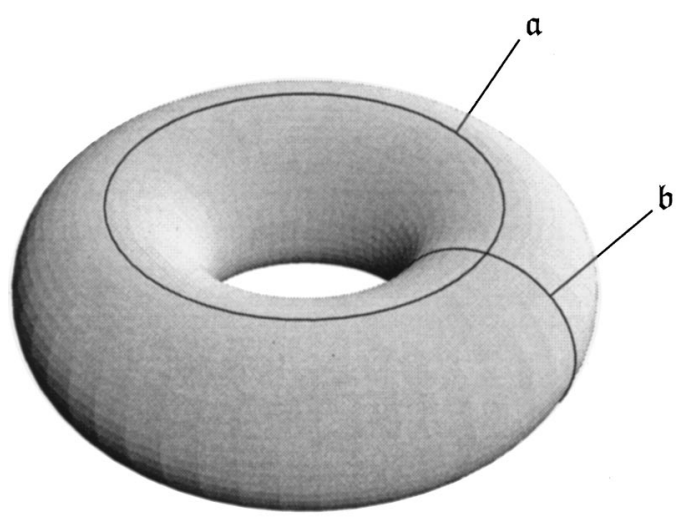

FIG. 3. The torus, handlebody of genus 1, is topologically equivalent to the Riemann surface in Fig. 2. It has been made simply connected by introducing the dissections $\mathfrak{a}$ and $\mathfrak{b}$. 
simply connected, a key requirement in order for Cauchy's theorem to apply, and this is more easily appreciated on the dissected torus in Fig. 3. Examination of the integral in (27) shows that only $\mathcal{C}_{\mathfrak{a} \cup \mathfrak{b}}$, the portion of the path enclosing the branch cuts and dissections, provides a contribution. The rest of the integral vanishes either by symmetry, as for the parts along $\operatorname{Re} \alpha= \pm \pi$ on $\mathcal{C}_{ \pm \pi}$, or identically, as in the case where $|\operatorname{Im} \alpha|= \pm \infty$ on $\mathcal{C}_{ \pm \infty}$. Evaluation of the integral along $\mathcal{C}_{\mathfrak{a} \cup \mathfrak{b}}$ produces $^{11,12}$

$$
\int_{\mathcal{C}_{\mathfrak{a} \cup \mathfrak{b}}} V_{2 \pi}^{1}(\alpha, u) d V_{2 \pi}^{3}(\alpha, u)=\mathfrak{A}_{2 \pi}^{1} \mathfrak{B}_{2 \pi}^{3}-\mathfrak{B}_{2 \pi}^{1} \mathfrak{A}_{2 \pi}^{3}
$$

where, extending our notation, capitalized letters denote cyclic periods on cycles identified by the correspond lower case letters so that, for example, $\mathfrak{A}_{2 \pi}^{1}=\int_{\mathfrak{a}} v_{2 \pi}^{1} d \alpha$. The cycles defined by the dissections $\mathfrak{a}$ and $\mathfrak{b}$ in Fig. 2 are the same as the cycles $a$ and $b$ shown in Fig. 1 so that $\mathfrak{A}_{2 \pi}^{n}$ $=A_{2 \pi}^{n}$ and $\mathfrak{B}_{2 \pi}^{n}=B_{2 \pi}^{n}$. We therefore obtain, in light of (26), the remarkable result

$$
\int_{\mathcal{C}_{1}} V_{2 \pi}^{1}(\alpha, u) d V_{2 \pi}^{3}(\alpha, u)=\mathfrak{A}_{2 \pi}^{1} \mathfrak{B}_{2 \pi}^{3}-\mathfrak{B}_{2 \pi}^{1} \mathfrak{A}_{2 \pi}^{3}=A_{2 \pi}^{1} B_{2 \pi}^{3}-B_{2 \pi}^{1} A_{2 \pi}^{3}
$$

which, by virtue of (27), can be expressed as a sum of residues. On the Riemann surface, the residues of the integrand in (27) are given by

$$
\begin{aligned}
& -\left.\frac{u\left(\zeta_{2 \pi}\right)}{u(\alpha)} \frac{\cos \alpha}{\cos \zeta_{2 \pi}} \frac{\sin \zeta_{2 \pi}}{\sin \alpha} \int_{(\eta, 0)}^{(\alpha, u)} v_{2 \pi}^{1}\left(\alpha^{\prime}\right) d \alpha^{\prime}\right|_{\alpha=\left( \pm \zeta_{2 \pi}, \pm u\right)} \\
& \quad= \begin{cases}\mp \int_{(\eta, 0)}^{(\alpha, u)} v_{2 \pi}^{1}\left(\alpha^{\prime}\right) d \alpha^{\prime}, & \alpha=\left(\zeta_{2 \pi}, \pm u\right), \\
\pm \int_{(\eta, 0)}^{(\alpha, u)} v_{2 \pi}^{1}\left(\alpha^{\prime}\right) d \alpha^{\prime}, & \alpha=\left(-\zeta_{2 \pi}, \pm u\right),\end{cases}
\end{aligned}
$$

and these, after carrying out the integration on the dissected Riemann surface, can be expressed in terms of the elliptic integral $V_{2 \pi}^{1}(\alpha)$ defined on the upper Riemann sheet. Being mindful of the dissections and exploiting the numerous symmetries involved, we obtain

$$
\sum \operatorname{Res}=-A_{2 \pi}^{1} \pm 2 B_{2 \pi}^{1}-4 V_{2 \pi}^{1}\left(\zeta_{2 \pi}\right), \quad V_{2 \pi}^{1}\left(\zeta_{2 \pi}\right) \in\left\{-\tau^{\prime} \frac{A_{2 \pi}^{1}}{4} \pm \tau \frac{B_{2 \pi}^{1}}{2}: 0 \leqslant \tau^{\prime}, \tau \leqslant 1\right\} .
$$

It would of course be impossible to obtain a unique expression for the above if the Riemann surface had not previously been made simply connected. Substitution of (28) and (29) in (27) yields

$$
V_{2 \pi}^{1}\left(\zeta_{2 \pi}\right)=-\frac{A_{2 \pi}^{1}}{4} \pm \frac{B_{2 \pi}^{1}}{2}-\frac{\sigma_{2 \pi}}{8 \pi i}\left(A_{2 \pi}^{1} B_{2 \pi}^{3}-B_{2 \pi}^{1} A_{2 \pi}^{3}\right)=-\frac{A_{2 \pi}^{1}}{4} \pm \frac{B_{2 \pi}^{1}}{2}+\sigma_{2 \pi} i \Lambda_{2 \pi}
$$

where the only unknown is $\zeta_{2 \pi}$ and we have reintroduced $\sigma_{2 \pi}$ from Eq. (20). The quantity $\Lambda_{2 \pi}$ is, from (26), defined as

$$
\Lambda_{2 \pi}=\frac{1}{16 \pi}\left\{A_{2 \pi}^{1}\left(-B_{4 \pi}^{3}+D_{4 \pi}^{3}-B_{0+1}+D_{0+1}\right)-B_{2 \pi}^{1}\left(-\kappa_{4 \pi} A_{4 \pi}^{1}-A_{4 \pi}^{3}+C_{4 \pi}^{3}-A_{0+1}+C_{0+1}\right)\right\} .
$$

This is a known quantity provided equation system (23) has been solved, a procedure carried out in the next section. 


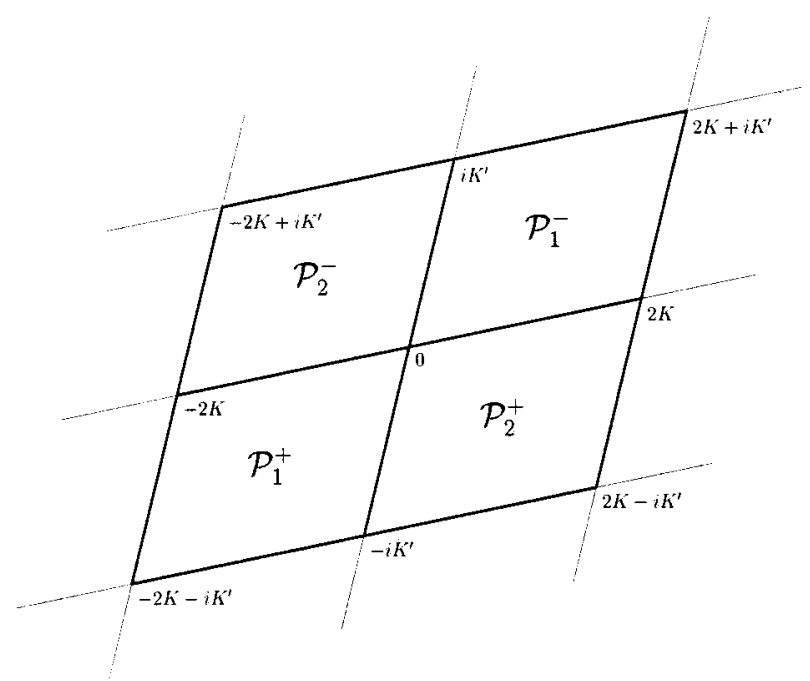

FIG. 4. The regions $\mathcal{P}_{1}^{ \pm}$and $\mathcal{P}_{2}^{ \pm}$in terms of the complete integrals of the first kind $K$ and $K^{\prime}$ with $k=\cos \eta$. The parallelograms $\mathcal{P}$ indicate the various ranges in which $\Lambda_{2 \pi}$ must lie when carrying out the inversion for $\zeta_{2 \pi}$ with Eq. (34).

A closed-form expression for $\zeta_{2 \pi}$ can be found by using the Jacobian elliptic sine function sn to invert the elliptic integral of the first kind in (30). Legendre's standard form for the integral is

$$
F(x, k)=\int_{0}^{x} \frac{d t}{\sqrt{\left(1-t^{2}\right)\left(1-k^{2} t^{2}\right)}},
$$

where $k$ is the modulus of the integral and the elliptic sine function is such that

$$
\operatorname{sn}[F(x, k), k]=x
$$

with $x$ located in the fundamental period parallelogram of dimensions $4 K$ and $2 i K^{\prime}$ centered at the origin of the complex plane. The periods $K$ and $K^{\prime}$ are the standard quantities defined, respectively, by the complete elliptic integrals of the first kind with modulus $k$ and complementary modulus $k^{\prime}$. The transformation $x=\cos \alpha / \cos \eta$ then shows that

$$
V_{2 \pi}^{1}(\alpha)=\int_{\eta}^{\alpha} \frac{d \alpha^{\prime}}{\sqrt{\cos ^{2} \alpha^{\prime}-\cos ^{2} \eta}}=i\left\{F\left(\frac{\cos \alpha}{\cos \eta}, \cos \eta\right)+i \frac{B_{2 \pi}^{1}}{4}\right\}
$$

and we note the following useful relationships:

$$
k=\cos \eta, \quad A_{2 \pi}^{1}=4 K^{\prime}, \quad B_{2 \pi}^{1}=4 i K .
$$

Finally, taking into account the definition of $\Sigma$ Res in terms of the range of $V_{2 \pi}^{1}\left(\zeta_{2 \pi}\right)$ given in (29) and inserting (32) in (30), we obtain after some algebraic manipulations

$$
\zeta_{2 \pi}=\left\{\begin{array}{cc}
\arccos \left[k \operatorname{sn}\left(i K^{\prime}+3 K+\sigma_{2 \pi} \Lambda_{2 \pi}, k\right)\right], & \Lambda_{2 \pi} \in \mathcal{P}_{1}^{\sigma_{2 \pi}}, \\
\arccos \left[k \operatorname{sn}\left(i K^{\prime}-K+\sigma_{2 \pi} \Lambda_{2 \pi}, k\right)\right], & \Lambda_{2 \pi} \in \mathcal{P}_{2}^{\sigma_{2 \pi}},
\end{array}\right.
$$

which is an explicit expression for $\zeta_{2 \pi}$. The correct expression to use in (34) as well as the correct definition for $\sigma_{2 \pi}= \pm 1$ follow from locating $\Lambda_{2 \pi}$ in the appropriate $\mathcal{P}$ parallelogram in Fig. 4. For instance, if $\Lambda_{2 \pi} \in \mathcal{P}_{2}^{-}$, then $\sigma_{2 \pi}=-1$ and $\zeta_{2 \pi}=\arccos \left[k \operatorname{sn}\left(i K^{\prime}-K-\Lambda_{2 \pi}, k\right)\right]$. The multiplicative constant $\kappa_{2 \pi}$ follows immediately from (25a) or (25b). The period parallelograms in Fig. 4 were obtained by using (30) and (29) to specify the range of $\Lambda_{2 \pi}$ in terms of that of $V_{2 \pi}^{1}\left(\zeta_{2 \pi}\right)$. For 


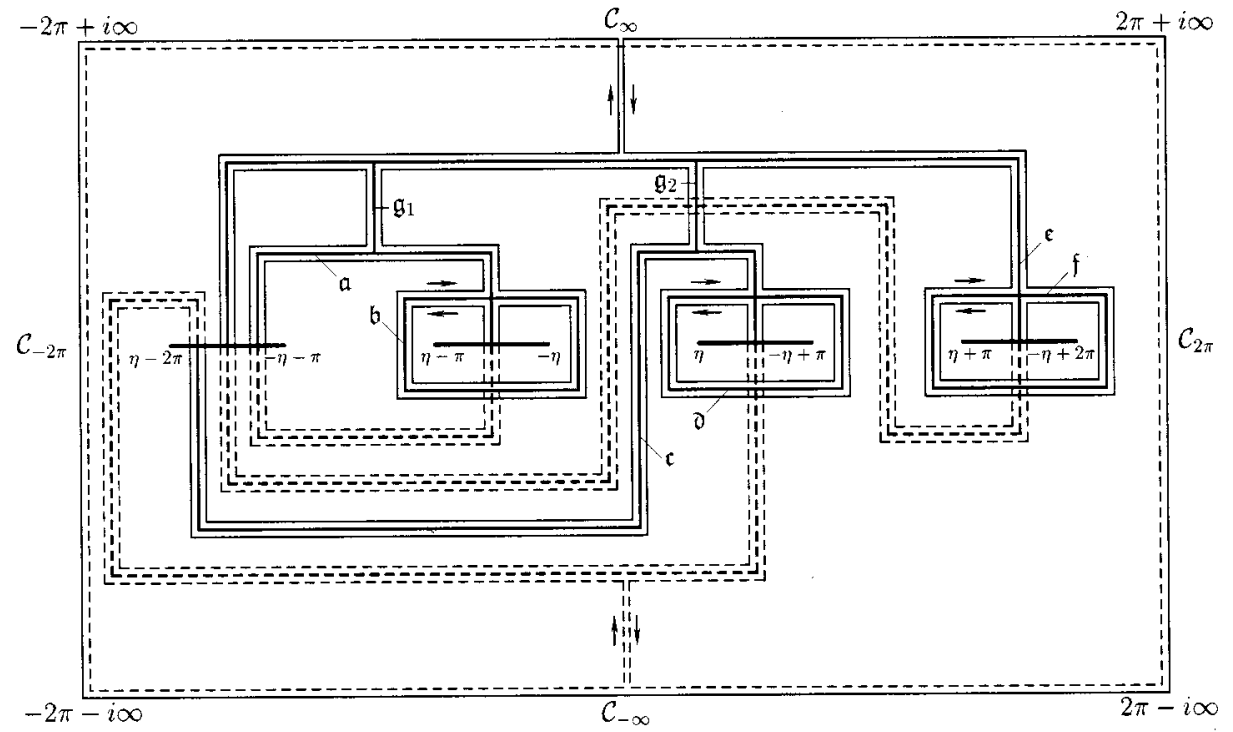

FIG. 5. The contour $\mathcal{C}_{2}=\mathcal{C}_{\mathfrak{a} \cup \mathfrak{b}} \cup \mathcal{C}_{\mathfrak{c} \cup \supset} \cup \mathcal{C}_{\mathfrak{e} \cup \mathfrak{f}} \cup \mathcal{C}_{\mathfrak{g}_{1,2}} \cup \mathcal{C}_{ \pm 2 \pi} \cup \mathcal{C}_{ \pm \infty}$ on the upper (solid line) and lower (dashed line) Riemann sheets. The thicker inner lines are the dissections $\mathfrak{a}, \mathfrak{b}, \mathfrak{c}, \mathfrak{d}, \mathfrak{e}, \mathfrak{f}$ and $\mathfrak{g}_{1,2}$ introduced to make the Riemann surface simply connected. The path $\mathcal{C}_{\mathfrak{a} \cup \mathfrak{b}}$ denotes, for example, the portion of the contour enclosing the dissecting cycles $\mathfrak{a}$ and $\mathfrak{b}$.

example, if we consider the case of $+2 B_{2 \pi}^{1}$, then it follows that $\sigma_{2 \pi} \Lambda_{2 \pi} \in[-2 K, 0]$ $\times\left[-i K^{\prime}, 0\right]$ and, since $\sigma_{2 \pi}= \pm 1$, this corresponds to the requirement that $\Lambda_{2 \pi}$ lies in either of the parallelograms $\mathcal{P}_{1}^{\sigma_{2 \pi}}$ shown in Fig. 4. One proceeds similarly for the case where we have $-2 B_{2 \pi}^{1}$ to obtain the $\mathcal{P}_{2}^{\sigma_{2 \pi}}$ parallelograms in the figure. Taking into account the periodicity of the elliptic sine function, (34) becomes

$$
\zeta_{2 \pi}=\arccos \left[k \operatorname{sn}\left(i K^{\prime}+3 K+\sigma_{2 \pi} \Lambda_{2 \pi}, k\right)\right], \quad \Lambda_{2 \pi} \in \mathcal{P}_{1}^{\sigma_{2 \pi}} \cup \mathcal{P}_{2}^{\sigma_{2 \pi}}
$$

where $\Lambda_{2 \pi}$ is given in (31) and the standard periods $K$ and $i K^{\prime}$ (as well as the parameter $k$ ) are given in (33).

\section{DETERMINATION OF $\zeta_{4 \pi}$ AND $\kappa_{4 \pi}$}

A similar procedure to the one given in the previous section is required to successfully invert for $\zeta_{4 \pi}$ in (24). However, the cyclic periods appearing on the left-hand side of (24) are now related to $4 \pi$ periodic expressions and the application of Cauchy's theorem must now be carried out on the Riemann surface delimited by the contour $\mathcal{C}_{2}$ of width $4 \pi$ shown in Fig. 5. Proceeding as in Sec. III we consider

$$
\int_{\mathcal{C}_{2}} V_{4 \pi}^{1}(\alpha, u) d V_{4 \pi}^{3}(\alpha, u)=2 \pi i \sum \operatorname{Res},
$$

with the elliptic integrals defined as in the previous section but using the $4 \pi$ periodic terms $v_{4 \pi}^{1,3}(\alpha, u)$. For $4 \pi$ periodic functions, the enclosed surface is now the topological equivalent of a handlebody of genus three (a sphere with three handles) as shown in Fig. 6 and it can be appreciated that making it simply connected involves a larger number of dissections than the torus of the previous section. To make it so, three pairs of canonical dissections $\mathfrak{a}, \mathfrak{b} ; \mathfrak{c}, \mathfrak{d}$ and $\mathfrak{e}, \mathfrak{f}$ are required as well as the two auxiliary dissections $\mathfrak{g}_{1}$ and $\mathfrak{g}_{2}$. They are shown in both Figs. 5 and 6; the simple connectedness is once again better appreciated by examining the handlebody representation of the Riemann surface. To keep the analysis relatively straightforward it is beneficial to draw the dissections such that only members of dissection pairs, $\mathfrak{a}$ and $\mathfrak{b}$, for example, intersect. This, 


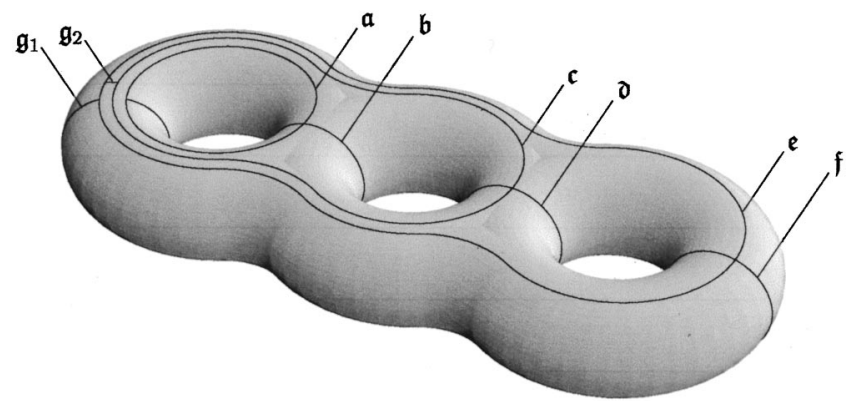

FIG. 6. Handlebody of genus 3, the topological equivalent of the Riemann surface in Fig. 5. It has been made simply connected by introducing the dissections $\mathfrak{a}, \mathfrak{b}, \mathfrak{c}, \mathfrak{d}, \mathfrak{e}, \mathfrak{f}$ and $\mathfrak{g}_{1,2}$.

while simplifying the evaluation of the path integral around the dissections, entails the rather intricate set of dissections shown in Fig. 5. Carrying out the integration it is seen that only $\mathcal{C}_{\mathfrak{a} \cup \mathfrak{b}} \cup \mathcal{C}_{\mathfrak{c} \cup \mathfrak{d}} \cup \mathcal{C}_{\mathfrak{e} \cup \mathfrak{f}}$, the portion of the path enclosing the dissection pairs, contributes. The rest of the integral vanishes either by symmetry, as for the parts along $\operatorname{Re} \alpha= \pm 2 \pi$ on $\mathcal{C}_{ \pm 2 \pi}$, or identically, as in the case where $|\operatorname{Im} \alpha|= \pm \infty$ on $\mathcal{C}_{ \pm \infty}$. The contributions from the path enclosing the three dissection pairs, following our previous work, are

$$
\begin{aligned}
& \int_{\mathcal{C}_{\mathfrak{a} \cup \mathfrak{b}}} V_{4 \pi}^{1}(\alpha, u) d V_{4 \pi}^{3}(\alpha, u)=\mathfrak{A}_{4 \pi}^{1} \mathfrak{B}_{4 \pi}^{3}-\mathfrak{B}_{4 \pi}^{1} \mathfrak{A}_{4 \pi}^{3}, \\
& \int_{\mathcal{C}_{\mathfrak{c} \cup \mathfrak{d}}} V_{4 \pi}^{1}(\alpha, u) d V_{4 \pi}^{3}(\alpha, u)=\mathfrak{C}_{4 \pi}^{1} \mathfrak{D}_{4 \pi}^{3}-\mathfrak{D}_{4 \pi}^{1} \mathfrak{C}_{4 \pi}^{3}, \\
& \int_{\mathcal{C}_{\mathrm{e} \cup f}} V_{4 \pi}^{1}(\alpha, u) d V_{4 \pi}^{3}(\alpha, u)=\mathfrak{E}_{4 \pi}^{1} \mathfrak{F}_{4 \pi}^{3}-\mathfrak{F}_{4 \pi}^{1} \mathfrak{E}_{4 \pi}^{3} .
\end{aligned}
$$

Comparing the canonical cycles defined by the dissections with those defined in Fig. 1, together with symmetry, it is possible to rewrite the above canonical periods in terms of the cyclic periods defined in (19). The cyclic periods are defined on intervals between adjacent branch points and extending these definitions to the negative real axis, by means of the even parity of the expressions, the contributions from the branch point to branch point integrals in $\mathcal{S}_{4 \pi}$ can then be identified as shown in Fig. 7(a). The canonical cycles $\mathfrak{a}, \mathfrak{b}, \mathfrak{c}, \mathfrak{d}, \mathfrak{e}$ and $\mathfrak{f}$ from Fig. 5 are then partitioned into branch point to branch point contributions, as shown in Figs. 7(b)-7(d). By comparing with Fig. 7(a), they are easily expressed in terms of the cyclic periods and it can thus be shown that

$$
\begin{gathered}
\mathfrak{A}=C, \quad \mathfrak{B}=-B, \\
\mathfrak{C}=A+B+C+D, \quad \mathfrak{D}=B, \\
\mathfrak{E}=A+B+2 C, \quad \mathfrak{F}=D .
\end{gathered}
$$

This yields the following equalities for the right-hand sides of the above equations:

$$
\begin{gathered}
\mathfrak{A}_{4 \pi}^{1} \mathfrak{B}_{4 \pi}^{3}-\mathfrak{B}_{4 \pi}^{1} \mathfrak{A}_{4 \pi}^{3}=B_{4 \pi}^{1} C_{4 \pi}^{3}-C_{4 \pi}^{1} B_{2 \pi}^{3}, \\
\mathfrak{C}_{4 \pi}^{1} \mathfrak{D}_{4 \pi}^{3}-\mathfrak{C}_{4 \pi}^{1} \mathfrak{D}_{4 \pi}^{3}=A_{4 \pi}^{1} B_{4 \pi}^{3}-B_{4 \pi}^{1} A_{4 \pi}^{3}+C_{4 \pi}^{1} B_{4 \pi}^{3}-B_{4 \pi}^{1} C_{4 \pi}^{3}+D_{4 \pi}^{1} B_{4 \pi}^{3}-B_{4 \pi}^{1} D_{4 \pi}^{3}, \\
\mathfrak{E}_{4 \pi}^{1} \mathfrak{F}_{4 \pi}^{3}-\mathfrak{E}_{4 \pi}^{1} \mathfrak{F}_{4 \pi}^{3}=A_{4 \pi}^{1} D_{4 \pi}^{3}-D_{4 \pi}^{1} A_{4 \pi}^{3}+B_{4 \pi}^{1} D_{4 \pi}^{3}-D_{4 \pi}^{1} B_{4 \pi}^{3}+2\left(C_{4 \pi}^{1} D_{4 \pi}^{3}-D_{4 \pi}^{1} C_{4 \pi}^{3}\right) .
\end{gathered}
$$




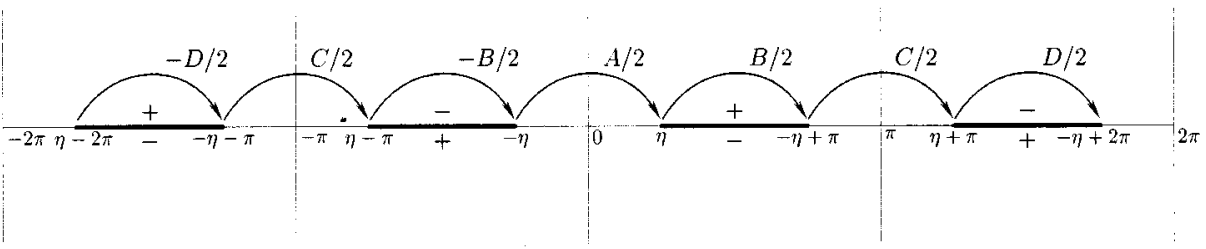

(a) Basic cyclic periods

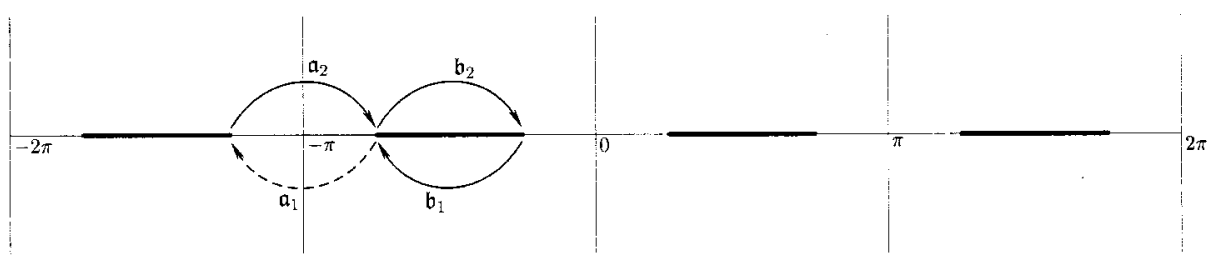

(b) Canonical cycles $\mathfrak{a}$ and $\mathfrak{b}$

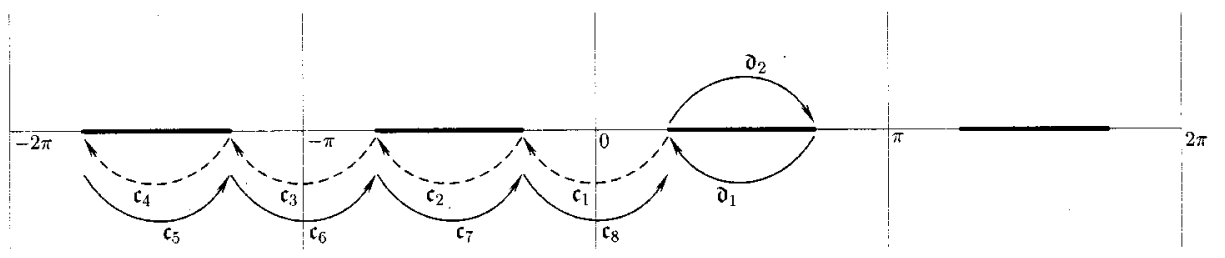

(c) Canonical cycles $\mathfrak{c}$ and $\mathfrak{a}$

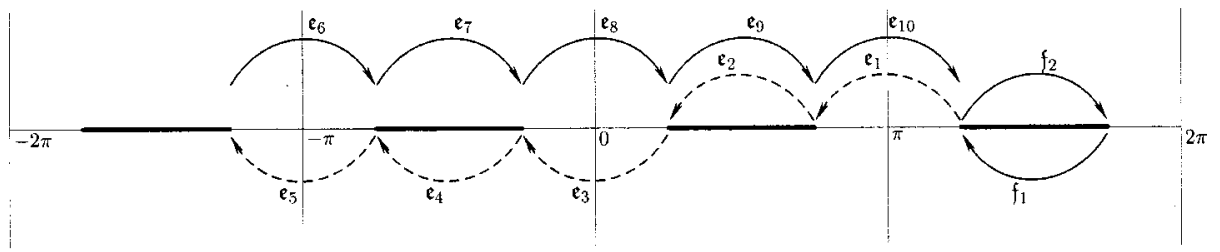

(d) Canonical cycles $\mathfrak{e}$ and $\mathfrak{f}$

FIG. 7. Figures used to express the canonical periods in Fig. 5 in terms of the basic cyclic periods in Fig. 1 (e.g., $\mathfrak{A}$ in terms of $A, B, C, D$ ). The canonical cycles on the (solid lines) upper and (dashed lines) lower sheets in (b), (c) and (d) are written as chains of branch point to branch point segments- $\mathfrak{e}$ into a sequence of $\mathfrak{e}_{n}$, for example-which are easily expressed in terms of the basic cyclic periods given in (a).

Summing up these contributions,

$$
\begin{aligned}
\int_{\mathcal{C}_{2}} V_{4 \pi}^{1}(\alpha, u) d V_{4 \pi}^{3}(\alpha, u) & =\left(\int_{\mathcal{C}_{\mathrm{a} \cup \mathfrak{b}}}+\int_{\mathcal{C}_{\mathfrak{c} \cup \mathcal{0}}}+\int_{\mathcal{C}_{\mathfrak{e} \cup f}}\right) V_{4 \pi}^{1}(\alpha, u) d V_{4 \pi}^{3}(\alpha, u) \\
& =A_{4 \pi}^{1} B_{4 \pi}^{3}-B_{4 \pi}^{1} A_{4 \pi}^{3}+A_{4 \pi}^{1} D_{4 \pi}^{3}-D_{4 \pi}^{1} A_{4 \pi}^{3}+2\left(C_{4 \pi}^{1} D_{4 \pi}^{3}-D_{4 \pi}^{1} C_{4 \pi}^{3}\right) \\
& =A_{4 \pi}^{1}\left(B_{4 \pi}^{3}+D_{4 \pi}^{3}\right)-2 B_{4 \pi}^{1}\left(A_{4 \pi}^{3}+C_{4 \pi}^{3}\right),
\end{aligned}
$$

where on the last line we have made use of $D_{4 \pi}^{1}=B_{4 \pi}^{1}$ and $C_{4 \pi}^{1}=0$. This is remarkable in that it reproduces the left-hand side of (24) and can be expressed in terms of residues in accordance with (36). The integrand in (36) has residues 


$$
\begin{aligned}
& -\left.\frac{u\left(\zeta_{4 \pi}\right)}{u(\alpha)} \frac{\cos \alpha}{\cos \zeta_{4 \pi}} \frac{\sin \left(\zeta_{4 \pi} / 2\right)}{\sin (\alpha / 2)} \int_{(\eta, 0)}^{(\alpha, u)} v_{4 \pi}^{1}\left(\alpha^{\prime}\right) d \alpha^{\prime}\right|_{\alpha=\left( \pm \zeta_{4 \pi}, \pm u\right)} \\
& = \begin{cases}\mp \int_{(\eta, 0)}^{(\alpha, u)} v_{4 \pi}^{1}\left(\alpha^{\prime}\right) d \alpha^{\prime}, & \alpha=\left(\zeta_{4 \pi}, \pm u\right), \\
\pm \int_{(\eta, 0)}^{(\alpha, u)} v_{4 \pi}^{1}\left(\alpha^{\prime}\right) d \alpha^{\prime}, & \alpha=\left(-\zeta_{4 \pi}, \pm u\right),\end{cases}
\end{aligned}
$$

which are expressed in terms of $V_{4 \pi}^{1}\left(\zeta_{4 \pi}\right)$ and $V_{4 \pi}^{1}\left(2 \pi-\zeta_{4 \pi}\right)$-both taken on the top Riemann sheet - after carrying out the path integrals on the dissected Riemann surface. Taking advantage of the numerous symmetries and avoiding the crossing of any dissection leads to

$$
\sum \operatorname{Res}= \begin{cases}-A_{4 \pi}^{1} \pm 4 B_{4 \pi}^{1}-4 V_{4 \pi}^{1}\left(\zeta_{4 \pi}\right), & V_{4 \pi}^{1}\left(\zeta_{4 \pi}\right) \in\left\{-\tau^{\prime} \frac{A_{4 \pi}^{1}}{4} \pm \tau \frac{B_{4 \pi}^{1}}{2}: 0 \leqslant \tau^{\prime}, \tau \leqslant 1\right\}, \\ -A_{4 \pi}^{1}-4 V_{4 \pi}^{1}\left(2 \pi-\zeta_{4 \pi}\right), & V_{4 \pi}^{1}\left(2 \pi-\zeta_{4 \pi}\right) \in\left\{-\tau^{\prime} \frac{A_{4 \pi}^{1}}{4} \pm \tau \frac{B_{4 \pi}^{1}}{2}: 0 \leqslant \tau^{\prime}, \tau \leqslant 1\right\} .\end{cases}
$$

In the short analysis that follows, we restrict for now our attention to the first case given above with the $+4 B_{4 \pi}^{1}$ term for the sake of brevity. Using (40) and (41) in (36) then produces

$$
\begin{aligned}
V_{4 \pi}^{1}\left(\zeta_{4 \pi}\right) & =-\frac{A_{4 \pi}^{1}}{4}+B_{4 \pi}^{1}-\frac{\sigma_{4 \pi}}{8 \pi i}\left\{A_{4 \pi}^{1}\left(B_{4 \pi}^{3}+D_{4 \pi}^{3}\right)-2 B_{4 \pi}^{1}\left(A_{4 \pi}^{3}+C_{4 \pi}^{3}\right)\right\} \\
& =-\frac{A_{4 \pi}^{1}}{4}+B_{4 \pi}^{1}+\frac{\sigma_{4 \pi}}{\cos (\eta / 2)} \Lambda_{4 \pi}
\end{aligned}
$$

where the only unknown is $\zeta_{4 \pi}$ and the sign $\sigma_{4 \pi}$ from Eq. (20) has been reintroduced. The cos $\eta / 2$ term is used for future convenience and, in agreement with (24), we have

$$
\Lambda_{4 \pi}=\frac{\cos (\eta / 2)}{8 \pi i}\left\{A_{4 \pi}^{1}\left(B_{0+1}+D_{0+1}\right)-2 B_{4 \pi}^{1}\left(A_{0+1}+C_{0+1}\right)\right\} .
$$

In order to use the Jacobian elliptic sine function sn to invert the elliptic integral of the first kind, we recast $V_{4 \pi}^{1}(\alpha)$ in terms of Legendre's standard form. The transformation $x$ $=(\sin \alpha / 2) /(\sin \eta / 2)$, together with the alternative expression to $(6)$,

$$
u(\alpha)=2 \sqrt{\left(\cos ^{2} \frac{\alpha}{2}-\cos ^{2} \frac{\eta}{2}\right)\left(\cos ^{2} \frac{\alpha}{2}-\sin ^{2} \frac{\eta}{2}\right)},
$$

enables us to write

$$
V_{4 \pi}^{1}(\alpha)=\int_{\eta}^{\alpha} \frac{\cos \left(\alpha^{\prime} / 2\right)}{u\left(\alpha^{\prime}\right)} d \alpha^{\prime}=\frac{1}{\cos (\eta / 2)}\left\{F\left(\frac{\sin (\alpha / 2)}{\sin (\eta / 2)}, \tan \frac{\eta}{2}\right)-\frac{\cos (\eta / 2)}{4} A_{4 \pi}^{1}\right\},
$$

and we have the following relationships for the parameter $k$ and the cyclic periods:

$$
k=\tan \frac{\eta}{2}, \quad A_{4 \pi}^{1}=\frac{4 K}{\cos (\eta / 2)}, \quad B_{4 \pi}^{1}=\frac{2 i K^{\prime}}{\cos (\eta / 2)} .
$$

Inserting (44) in (42), solving for $\zeta_{4 \pi}$ then produces, after some algebraic manipulations, 


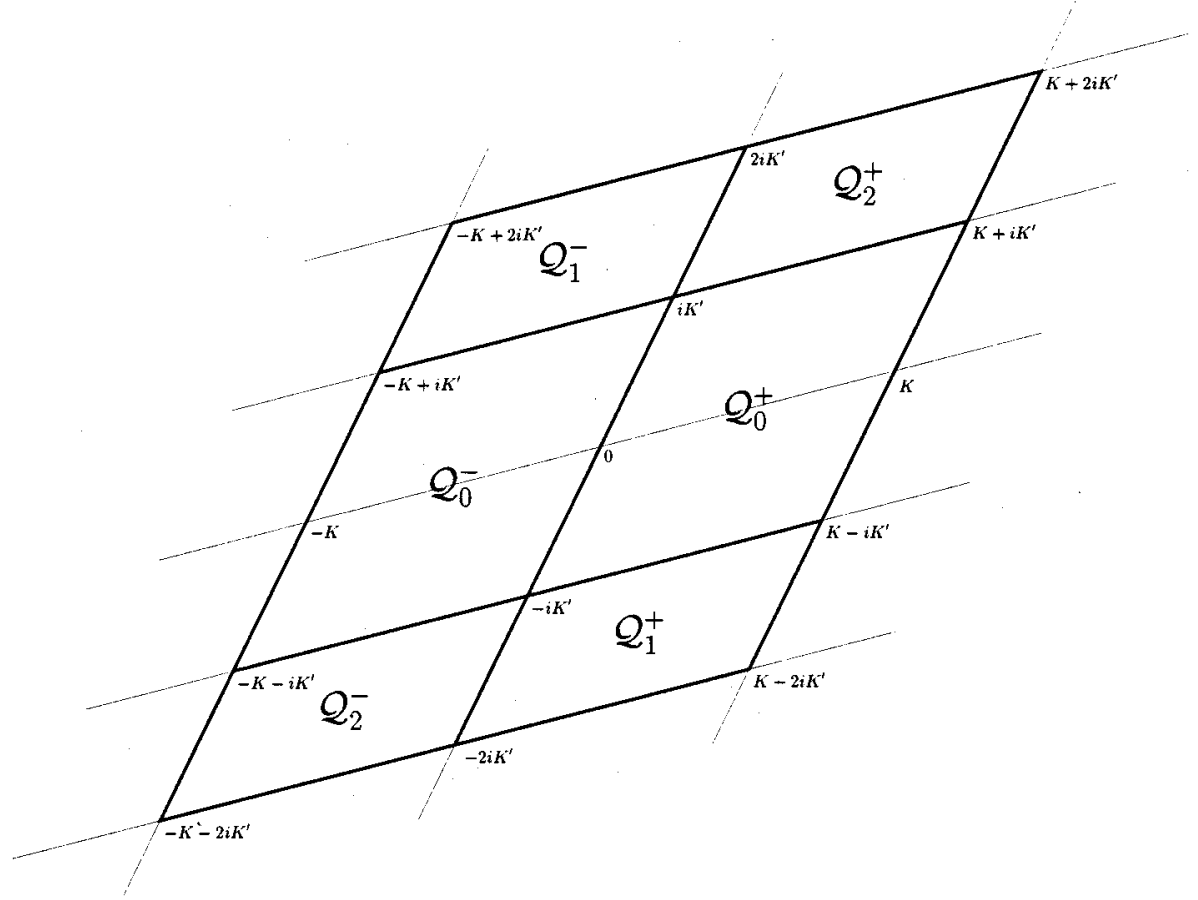

FIG. 8. The regions $\mathcal{Q}_{0}^{ \pm}, \mathcal{Q}_{1}^{ \pm}$and $\mathcal{Q}_{2}^{ \pm}$in terms of the complete integrals of the first kind $K$ and $K^{\prime}$ with $k=\tan \eta / 2$. The parallelograms $\mathcal{Q}$ indicate the various ranges in which $\Lambda_{4 \pi}$ must lie when carrying out the inversion for $\zeta_{4 \pi}$ with Eq. (46).

$$
\zeta_{4 \pi}=2 \arcsin \left[\sin \frac{\eta}{2} \operatorname{sn}\left(2 i K^{\prime}+\sigma_{4 \pi} \Lambda_{4 \pi}, k\right)\right] .
$$

It follows from (42) and (41) that this holds if $\sigma_{4 \pi} \Lambda_{4 \pi}$ lies in the period parallelogram $[0, K]$ $\times\left[-i K^{\prime},-2 i K^{\prime}\right]$. As $\sigma_{4 \pi}= \pm 1$, the acceptable range for $\Lambda_{4 \pi}$ is therefore $\pm[0, K] \times \pm\left[-i K^{\prime}\right.$, $\left.-2 i K^{\prime}\right]$ with the plus sign corresponding to $\sigma_{4 \pi}=1$. These regions are identified in Fig. 8 by the period parallelograms $\mathcal{Q}_{1}^{\sigma_{4 \pi}}$. Repeating this process for the other cases in (41), it can be shown that

$$
\zeta_{4 \pi}= \begin{cases}2 \pi-2 \arcsin \left[\sin \frac{\eta}{2} \operatorname{sn}\left(\sigma_{4 \pi} \Lambda_{4 \pi}, k\right)\right], & \Lambda_{4 \pi} \in \mathcal{Q}_{0}^{\sigma_{4 \pi},} \\ 2 \arcsin \left[\sin \frac{\eta}{2} \operatorname{sn}\left(2 i K^{\prime}+\sigma_{4 \pi} \Lambda_{4 \pi}, k\right)\right], & \Lambda_{4 \pi} \in \mathcal{Q}_{1}^{\sigma_{4 \pi},} \\ 2 \arcsin \left[\sin \frac{\eta}{2} \operatorname{sn}\left(-2 i K^{\prime}+\sigma_{4 \pi} \Lambda_{4 \pi}, k\right)\right], & \Lambda_{4 \pi} \in \mathcal{Q}_{2}^{\sigma_{4 \pi}},\end{cases}
$$

which is an explicit expression for $\zeta_{4 \pi}$. The proper expression and the sign of $\sigma_{4 \pi}= \pm 1$ are selected by using Fig. 8 in order to determine in which period parallelogram $\mathcal{Q}$ lies the quantity $\Lambda_{4 \pi}$. The multiplicative constant $\kappa_{4 \pi}$ immediately follows from (23a) or (23b). Note that (46) can be simplified by exploiting the periodicity of the sn function to obtain

$$
\zeta_{4 \pi}=\left\{\begin{array}{l}
2 \pi-2 \arcsin \left[\sin \frac{\eta}{2} \operatorname{sn}\left(\sigma_{4 \pi} \Lambda_{4 \pi}, k\right)\right], \quad \Lambda_{4 \pi} \in \mathcal{Q}_{0}^{\sigma_{4 \pi},} \\
2 \arcsin \left[\sin \frac{\eta}{2} \operatorname{sn}\left(\sigma_{4 \pi} \Lambda_{4 \pi}, k\right)\right], \quad \Lambda_{4 \pi} \in \mathcal{Q}_{1}^{\sigma_{4 \pi}} \cup \mathcal{Q}_{2}^{\sigma_{4 \pi}},
\end{array}\right.
$$

where $\Lambda_{4 \pi}$ is given in (43) and $k, i K^{\prime}$ are given in (45). 


\section{BRANCH-FREE SOLUTIONS}

The determination of the unknowns $\zeta_{2 \pi, 4 \pi}$ and $\kappa_{2 \pi, 4 \pi}$ completes the definition of the solution to the first order difference equations $w(\alpha, u)$ as given in (20). It still has branch points and is therefore multivalued owing to the arbitrariness of the branch of $u(\alpha)$ but its integrand is now such that the path integral is single-valued on either of the Riemann sheets. Meromorphic solutions to the second order difference equation can now be obtained through the use of expressions (10). We recall, however, that solutions free of poles and zeros in the strip $\mathcal{S}_{4 \pi}$ are required and therefore seek to use specific linear combinations of (10) to finalize the construction of the

solutions. Knowledge of the poles and zeros of $w(\alpha, u)$ is required to successfully complete this endeavor and, since they arise solely due to $v_{2 \pi}^{3}(\alpha, u)$ and $v_{4 \pi}^{3}(\alpha, u)$, it is straightforward to show that in $\mathcal{S}_{4 \pi}$

$$
w(\alpha, \pm u) \sim\left\{\frac{\alpha+\zeta_{2 \pi}}{\alpha-\zeta_{2 \pi}} \frac{\alpha+\left(\zeta_{2 \pi}-2 \pi\right)}{\alpha-\left(\zeta_{2 \pi}-2 \pi\right)} \frac{\alpha+\zeta_{4 \pi}}{\alpha-\zeta_{4 \pi}}\right\}^{ \pm 1},
$$

where $\sigma_{2 \pi}=\sigma_{4 \pi}=1$ is assumed for simplicity. With this information in hand, the poles of any linear combinations involving (10) are easily determined. Zeros are by nature more elusive and we rely on knowledge of the limiting functions in order to determine their number as well as general location. The cancellation of the poles and zeros is also complicated by the order requirement on the solutions which must be $\mathcal{O}(1)$ as $|\operatorname{Im} \alpha| \rightarrow \infty$.

We first present an entirely analytic approach from which two independent solutions are obtained. They satisfy the analyticity requirements, recover the known solutions $\Psi_{1}(\alpha)$ and $1 / \Psi_{1}(\alpha)$ when $\eta \rightarrow \pi / 2$, and their only shortcoming is that they vanish as $\eta \rightarrow 0$, though knowledge of (13) circumvents this difficulty. In an effort to obtain expressions that also recover the known solution $\Psi_{2}(\alpha)$ when $\eta \rightarrow 0$, a number of approaches relying on numerically locating zeros were explored but, despite producing more desirable behaviors, they fail as $\eta \rightarrow 0$ due to inadmissible poles that arise in the strip of analyticity in that limit. An example of such an approach is provided here which nearly succeeds in recovering both limiting functions $\Psi_{1}(\alpha)$ and $\Psi_{2}(\alpha)$.

In the following, we use the primed functions $t_{n}^{\prime}(\alpha)$ to denote intermediate branch-free solutions of the second order difference equation (2) which still have undesired poles and zeros in the strip $\mathcal{S}_{4 \pi}$ whereas the unprimed functions $t_{n}(\alpha)$ denote the appropriate branch-free and pole/zerofree solutions.

\section{A. Analytical solution}

We proceed by constructing two meromorphic solutions of (2), $t_{1}^{\prime}(\alpha)$ and $t_{2}^{\prime}(\alpha)$, sharing a common pole at $\zeta_{4 \pi}$ but having distinct unknown zeros $\alpha_{1}$ and $\alpha_{2}$, and then use a linear combination to obtain an expression with a known pole/zero pair. Proceeding in a manner similar to the technique presented in Ref. 9, we write

$$
t_{1}^{\prime}(\alpha)=\frac{T_{2 \pi}(\alpha)}{2}\left\{\left(1+\frac{f_{1}(\alpha)}{u(\alpha)}\right) w(\alpha, u)+\left(1-\frac{f_{1}(\alpha)}{u(\alpha)}\right) w(\alpha,-u)\right\},
$$

a linear combination of the branch-free forms (10). The functions $f_{1}(\alpha)$ and $T_{2 \pi}(\alpha)$ are $4 \pi$ periodic; $f_{1}(\alpha)$ is a trigonometric polynomial used to introduce zeros at appropriate locations in the $\alpha$ plane while the external multiplicative function $T_{2 \pi}(\alpha)$ is a rational trigonometric function used to annul poles and zeros. We observe that the introduction of double zeros coincident with the poles of either $w(\alpha, u)$ or $w(\alpha,-u)$ produces, by (48), a simple zero in the term in curly braces above. Thus, if we require

$$
1-\frac{f_{1}(\alpha)}{u(\alpha)} \sim\left(\alpha+\zeta_{2 \pi}\right)^{2}\left(\alpha+\left(\zeta_{2 \pi}-2 \pi\right)\right)^{2}\left(\alpha+\zeta_{4 \pi}\right),
$$


then the second term in braces in (49) has simple zeros coinciding with those of $w(\alpha, u)$ at $\alpha$ $=-\zeta_{2 \pi}, 2 \pi-\zeta_{2 \pi}$ and is finite at $\alpha=-\zeta_{4 \pi}$. This implies

$$
\left(1+\frac{f_{1}(\alpha)}{u(\alpha)}\right) w(\alpha, u)+\left(1-\frac{f_{1}(\alpha)}{u(\alpha)}\right) w(\alpha,-u) \sim \frac{\alpha+\zeta_{2 \pi}}{\alpha-\zeta_{2 \pi}} \frac{\alpha+\left(\zeta_{2 \pi}-2 \pi\right)}{\alpha-\left(\zeta_{2 \pi}-2 \pi\right)} \frac{\alpha-\alpha_{1}}{\alpha-\zeta_{4 \pi}},
$$

where $\alpha_{1}$ is the unknown location of a zero in the $\mathcal{S}_{4 \pi}$ strip. While the exact location of $\alpha_{1}$ is not easily determined, its general location is known when $\eta$ is in the neighborhood of $\pi / 2$. Indeed, as $\eta \rightarrow \pi / 2$ we have $f_{1}(\alpha) / u(\alpha) \rightarrow 1$ and

$$
\frac{1}{2}\left\{\left(1+\frac{f_{1}(\alpha)}{u(\alpha)}\right) w(\alpha, u)+\left(1-\frac{f_{1}(\alpha)}{u(\alpha)}\right) w(\alpha,-u)\right\} \stackrel{\eta \rightarrow \pi / 2}{\rightarrow} w(\alpha, u),
$$

and we conclude, see (48), that when $\eta$ is in the neighborhood of $\pi / 2, \alpha_{1}$ is in the neighborhood of $-\zeta_{4 \pi}$. Choosing $T_{2 \pi}(\alpha)$ to eliminate the poles and zeros associated with $\zeta_{2 \pi}$ gives

$$
T_{2 \pi}(\alpha)=\frac{\tan \left(\zeta_{2 \pi} / 2\right)-\tan (\alpha / 2)}{\tan \left(\zeta_{2 \pi} / 2\right)+\tan (\alpha / 2)}
$$

so that

$$
t_{1}^{\prime}(\alpha) \sim \frac{\alpha-\alpha_{1}}{\alpha-\zeta_{4 \pi}}
$$

The $4 \pi$ periodic $f_{1}(\alpha)$ is obtained by letting

$$
f_{1}(\alpha)=\nu_{1}+\nu_{2} \cos \alpha+\nu_{3} \sin \alpha+\nu_{4} \cos \frac{\alpha}{2}+\nu_{5} \sin \frac{\alpha}{2}
$$

and enforcement of (50) produces

$$
\begin{gathered}
\nu_{1}=\frac{1}{1-\cos \left(\zeta_{2 \pi}-\zeta_{4 \pi}\right)}\left\{u\left(\zeta_{2 \pi}\right)-\frac{1}{u\left(\zeta_{4 \pi}\right)}\left(\sin ^{2} \eta \cos \zeta_{2 \pi} \cos \zeta_{4 \pi}-\cos ^{2} \eta \sin \zeta_{2 \pi} \sin \zeta_{4 \pi}\right)\right\}, \\
\nu_{2}=\cos \zeta_{2 \pi}\left(-\nu_{1}+\frac{\sin ^{2} \eta}{u\left(\zeta_{2 \pi}\right)}\right), \\
\nu_{3}=\sin \zeta_{2 \pi}\left(\nu_{1}+\frac{\cos ^{2} \eta}{u\left(\zeta_{2 \pi}\right)}\right),
\end{gathered}
$$

with $\nu_{4}=\nu_{5}=0$. We note that, in agreement with the analyticity requirements, the function $T_{2 \pi}(\alpha)$ and the ratio $f_{1}(\alpha) / u(\alpha)$ are $\mathcal{O}(1)$ as $|\operatorname{Im} \alpha| \rightarrow \infty$. A related meromorphic solution to the second order difference equation sharing the same pole but having a different zero is

$$
t_{2}^{\prime}(\alpha)=\frac{T_{2 \pi}(\alpha)}{2}\left\{\left(1-\frac{f_{2}(\alpha)}{u(\alpha)}\right) w(\alpha, u)+\left(1+\frac{f_{2}(\alpha)}{u(\alpha)}\right) w(\alpha,-u)\right\},
$$

where $f_{2}(\alpha)$ is now chosen, following the same kind of procedure as for $f_{1}(\alpha)$, such that

$$
\begin{gathered}
1-\frac{f_{2}(\alpha)}{u(\alpha)} \sim\left(\alpha-\zeta_{2 \pi}\right)^{2}\left(\alpha-\left(\zeta_{2 \pi}-2 \pi\right)\right)^{2}, \\
1+\frac{f_{2}(\alpha)}{u(\alpha)} \sim\left(\alpha+\zeta_{4 \pi}\right),
\end{gathered}
$$


and hence

$$
t_{2}^{\prime}(\alpha) \sim \frac{\alpha-\alpha_{2}}{\alpha-\zeta_{4 \pi}} .
$$

By the same reasoning as above, the zero $\alpha_{2}$ is also in the neighborhood of $\alpha=-\zeta_{4 \pi}$ when $\eta$ is in the neighborhood of $\pi / 2$. The meromorphic solutions $t_{1}^{\prime}(\alpha)$ and $t_{2}^{\prime}(\alpha)$ then share the same pole and a linear combination can now be used to introduce a zero at $\alpha=-\zeta_{4 \pi}$ such that

$$
t_{1}^{\prime}(\alpha)+\xi t_{2}^{\prime}(\alpha) \sim \frac{\alpha+\zeta_{4 \pi}}{\alpha-\zeta_{4 \pi}}
$$

and this requires

$$
\xi=-\frac{t_{1}^{\prime}\left(-\zeta_{4 \pi}\right)}{t_{2}^{\prime}\left(-\zeta_{4 \pi}\right)}=-T_{2 \pi}^{2}\left(-\zeta_{4 \pi}\right) \frac{\sin \zeta_{4 \pi} \cos \zeta_{4 \pi}-u\left(\zeta_{4 \pi}\right)\left(\nu_{2} \sin \zeta_{4 \pi}+\nu_{3} \cos \zeta_{4 \pi}\right)}{\sin \zeta_{4 \pi} \cos \zeta_{4 \pi}+u\left(\zeta_{4 \pi}\right)\left(\nu_{2} \sin \zeta_{4 \pi}+\nu_{3} \cos \zeta_{4 \pi}\right)} .
$$

An acceptable solution of the second order difference equation (2), free of poles and zeros in $\mathcal{S}_{4 \pi}$ and $\mathcal{O}(1)$ as $|\operatorname{Im} \alpha| \rightarrow \infty$, is then

$$
t_{1}(\alpha)=\frac{\tan \left(\zeta_{4 \pi} / 4\right)-\tan (\alpha / 4)}{\tan \left(\zeta_{4 \pi} / 4\right)+\tan (\alpha / 4)}\left\{t_{1}^{\prime}(\alpha)+\xi t_{2}^{\prime}(\alpha)\right\},
$$

and

$$
t_{1}(\alpha) \stackrel{\eta \rightarrow \pi / 2}{\rightarrow} \Psi_{1}(\alpha), \quad t_{1}(\alpha) \stackrel{\eta \rightarrow 0}{\rightarrow} 0 .
$$

The first limit stems from the fact that, as $\eta \rightarrow \pi / 2,1-f_{1}(\alpha) / u(\alpha) \rightarrow 0, \xi \rightarrow 0$ and, since in that limit $\kappa_{2 \pi, 4 \pi} \rightarrow 0$,

$$
w(\alpha, u) \stackrel{\eta \rightarrow \pi / 2}{\rightarrow} \frac{\tan \left(\zeta_{2 \pi} / 2\right)+\tan (\alpha / 2)}{\tan \left(\zeta_{2 \pi} / 2\right)-\tan (\alpha / 2)} \frac{\tan \left(\zeta_{4 \pi} / 4\right)+\tan (\alpha / 4)}{\tan \left(\zeta_{4 \pi} / 4\right)-\tan (\alpha / 4)} \Psi_{1}(\alpha),
$$

where we are still assuming that $\sigma_{2 \pi}=\sigma_{4 \pi}=1$. For the second limit, it is can be shown that $t_{1}^{\prime}$ $\rightarrow t_{2}^{\prime}$ and $\xi \rightarrow-1$ as $\eta \rightarrow 0$, and $t_{1}(\alpha)$ therefore vanishes in that limit. Following the same prescription as above, a second independent solution $t_{2}(\alpha)$ can be derived by seeking instead a common pole at $-\zeta_{4 \pi}$ and it can easily be shown that

$$
t_{2}(\alpha)=t_{1}(-\alpha),
$$

but we now have, using the same arguments as above,

$$
t_{2}(\alpha) \stackrel{\eta \rightarrow \pi / 2}{\rightarrow} \frac{1}{\Psi_{1}(\alpha)}, \quad t_{2}(\alpha) \stackrel{\eta \rightarrow 0}{\rightarrow} 0
$$

The pair of solutions $t_{1}(\alpha)$ and $t_{2}(\alpha)$ satisfy the prescribed analyticity requirements listed in Sec. I and recover the known solutions $\Psi_{1}(\alpha)$ and $1 / \Psi_{1}(\alpha)$ as $\eta \rightarrow \pi / 2$. However, both vanish as $\eta$ $\rightarrow 0$ and they therefore fall short of the preferred behavior obtained in Ref. 9 where the solutions are seen to vary smoothly as a function of $\eta$ between the two known limiting functions when $\eta$ $=\pi / 2$ and $\eta=0$. Although undesirable, this is not a serious shortcoming since a pair of linearly independent solutions are known when $\eta=0$ and are given in (13). This vanishing of $t_{1,2}(\alpha)$ when $\eta \rightarrow 0$ can be attributed to the use of linear combinations [see (52)] in order to create known zeros, a procedure which was not required in Ref. 9. Experience suggests that a purely multiplicative 


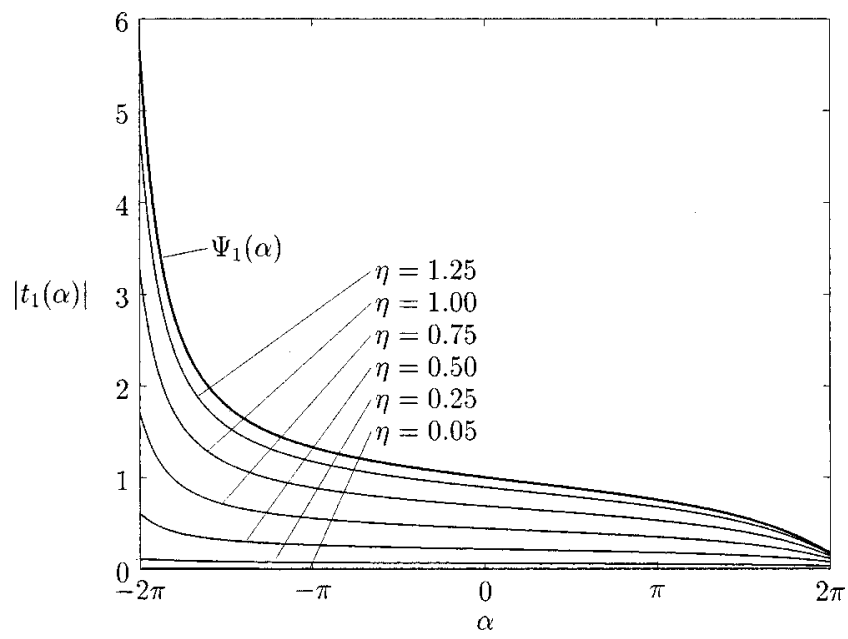

FIG. 9. Magnitude of the branch-free solution $t_{1}(\alpha)$ given in (53) when $\theta=0.25(1+i)$ for various values of $\eta$. The thicker line corresponds to the known limiting function $\Psi_{1}(\alpha)$, per (11), for $\eta=\pi / 2$. The case for $\eta=1.57$ is indistinguishable from $\Psi_{1}(\alpha)$.

method for eliminating the poles and zeros would likely reproduce the desired behavior, but this so far remains elusive. The continuation of $t_{1}(\alpha)$ and $t_{2}(\alpha)$ outside the strip $|\operatorname{Re} \alpha| \leqslant 2 \pi$ is provided by the first order difference equations (4) and (5), and the results confirm the fact that the solutions are free of branch points everywhere. Indeed, the expressions so obtained are linear combinations of the branch-free forms (10). The technique has been implemented and sample curves for $\left|t_{1}(\alpha)\right|$ are provided in Fig. 9 for various values of $\eta$ when $\theta=0.25(1+i)$. We observe that $t_{1}(\alpha)$ $\rightarrow \Psi_{1}(\alpha)$ as $\eta \rightarrow \pi / 2$, and $t_{1}(\alpha) \rightarrow 0$ as $\eta \rightarrow 0$.

We close this section by summarizing the procedure for computing the solutions $t_{1}(\alpha)$ and $t_{2}(\alpha)$. The fundamental building block is $w(\alpha, u)$, given in (20), the solution to the first order equation (4) and its computation requires the quantities $\kappa_{2 \pi, 4 \pi}, \zeta_{2 \pi, 4 \pi}$ and $\sigma_{2 \pi, 4 \pi}$. The preliminary step in obtaining those quantities is to first compute the cyclic periods (19) using numerical integration. The quantities $\zeta_{2 \pi}$ and $\sigma_{2 \pi}$ then follow from (35) and $\kappa_{2 \pi}$ from (25). The quantities $\zeta_{4 \pi}, \sigma_{4 \pi}$ and $\kappa_{4 \pi}$ are likewise obtained from (47) and (23). The function $w(\alpha, u)$ can then be computed by carrying out numerically the path integral in (20); the functions $t_{1}^{\prime}(\alpha), t_{2}^{\prime}(\alpha)$ and $t_{1}(\alpha), t_{2}(\alpha)$ then respectively follow from (49), (51) and (53), (54).

\section{B. Numerical solution}

By foregoing an entirely analytical approach, it is possible to construct solutions that, unlike $t_{1,2}(\alpha)$, do not vanish as $\eta \rightarrow 0$ but we must however resort to the numerical identification of zeros, a somewhat unattractive prospect. The main motivation behind this approach is to proceed with the pole/zero cancellation in a multiplicative fashion and avoid the use of linear combinations of the type (52). Interestingly, it is possible to reproduce in this manner the more desirable behavior obtained in Ref. 9 where Eq. (1) is solved. Therein, the solutions obtained are observed to smoothly vary between two known limiting functions corresponding to, in the case at hand, $\Psi_{1}(\alpha)$ as $\eta \rightarrow \pi / 2$ and $\Psi_{2}(\alpha)$ as $\eta \rightarrow 0$. This can be achieved here but at the price of having to numerically locate four zeros though, as discussed below, a pole that arises at $\eta \rightarrow 0$ proves to be problematic. Turning once again to the by now familiar form, we write

$$
t_{3}^{\prime}(\alpha)=\frac{T_{4 \pi}(\alpha)}{2}\left\{\left(1+\frac{f_{3}(\alpha)}{u(\alpha)}\right) w(\alpha, u)+\left(1-\frac{f_{3}(\alpha)}{u(\alpha)}\right) w(\alpha,-u)\right\},
$$

and examination of the behavior of the solution in Ref. 9 now suggests using 


$$
f_{3}(\alpha)=\frac{\cos \zeta_{4 \pi} \sin ^{2} \eta \cos \alpha+\sin \zeta_{4 \pi} \cos ^{2} \eta \sin \alpha}{u\left(\zeta_{4 \pi}\right)},
$$

so that

$$
1-\frac{f_{3}(\alpha)}{u(\alpha)} \sim\left(\alpha+\zeta_{4 \pi}\right)^{2}\left(\alpha+\left(\zeta_{4 \pi}-2 \pi\right)\right)^{2} .
$$

The term in braces in (55) is then such that

$$
\{\} \sim \frac{\alpha+\zeta_{4 \pi}}{\alpha-\zeta_{4}} \frac{\left(\alpha-\alpha_{1}\right)\left(\alpha-\alpha_{2}\right)\left(\alpha-\alpha_{3}\right)\left(\alpha-\alpha_{3}\right)}{\left(\alpha+\zeta_{2 \pi}\right)\left(\alpha-\zeta_{2 \pi}\right)\left(\alpha+\left(\zeta_{2 \pi}-2 \pi\right)\right)\left(\alpha+\left(\zeta_{2 \pi}+2 \pi\right)\right)}
$$

and the pole and zero associated with $\zeta_{4 \pi}$ are eliminated by choosing

$$
T_{4 \pi}(\alpha)=\frac{\tan \left(\zeta_{4 \pi} / 4\right)-\tan (\alpha / 4)}{\tan \left(\zeta_{4 \pi} / 4\right)+\tan (\alpha / 4)} .
$$

In the limit as $\eta \rightarrow \pi / 2$ we have

$$
t_{3}^{\prime}(\alpha) \sim \frac{\alpha+\zeta_{2 \pi}}{a-\zeta_{2 \pi}} \frac{\alpha+\left(\zeta_{2 \pi}-2 \pi\right)}{\alpha-\left(\zeta_{2 \pi}-2 \pi\right)},
$$

which implies that, when $\eta$ is in the neighborhood of $\pi / 2$, the poles at $\alpha=-\zeta_{2 \pi}$ and $\alpha=-\zeta_{2 \pi}$ $+2 \pi$ will each have a closely located pair of zeros $\alpha_{n}$. Once the location of these zeros has been obtained numerically by evaluating $t_{3}^{\prime}(\alpha)$ in (55), the desired solution may be written as

$$
t_{3}(\alpha)=\frac{\cos \alpha-\cos \zeta_{2 \pi}}{1-\cos \zeta_{2 \pi}}\left(\prod_{n=1}^{4} \frac{\sin \left(\alpha_{n} / 4\right)}{\sin \frac{1}{4}\left(\alpha_{n}-\alpha\right)}\right) t_{3}^{\prime}(\alpha)
$$

so that $t_{3}(\alpha)$ is free of poles and zeros in $\mathcal{S}_{4 \pi}$. It is easily shown that

$$
t_{3}(\alpha) \stackrel{\eta \rightarrow \pi / 2}{\rightarrow} \Psi_{1}(\alpha)
$$

and since $f_{3}(\alpha) / u(\alpha) \rightarrow \pm 1$ as $\eta \rightarrow 0$, albeit in a branched fashion, it can also be shown (numerically) that

$$
t_{3}(\alpha) \stackrel{\eta \rightarrow 0}{\rightarrow} \Psi_{2}(\alpha) .
$$

Figure 10 provides sample curves for $\left|t_{3}(\alpha)\right|$ for various values of $\eta$ when $\theta=0.25(1+i)$. The behavior obtained is reminiscent of the one in Ref. 9 since the solution now varies smoothly between the two limiting functions $\Psi_{1,2}(\alpha)$ as a function of $\eta$. Once again, a second solution is provided by $t_{3}(-\alpha)$ and this recovers $1 / \Psi_{1}(\alpha)$ as $\eta \rightarrow \pi / 2$ and $\Psi_{2}(\alpha)$ as $\eta \rightarrow 0$. Despite this, the approach is, however, flawed since one of the numerical zeros strays slightly outside $\mathcal{S}_{4 \pi}$ when $\eta \simeq 0.001$. This leads to failure of the solution in that limit since the corresponding zero canceling term in (56) gives rise to a pole within the strip of analyticity. It is unclear at this time if this is due to numerical inaccuracies or a fundamental limitation of the approach. It does, however, suggest that the construction procedure based on branch-free combinations of the function $w(\alpha, u)$ has the potential to recover solutions that vary smoothly between the two known limiting functions provided a proper method can be devised for constructing the branch-free solutions. 


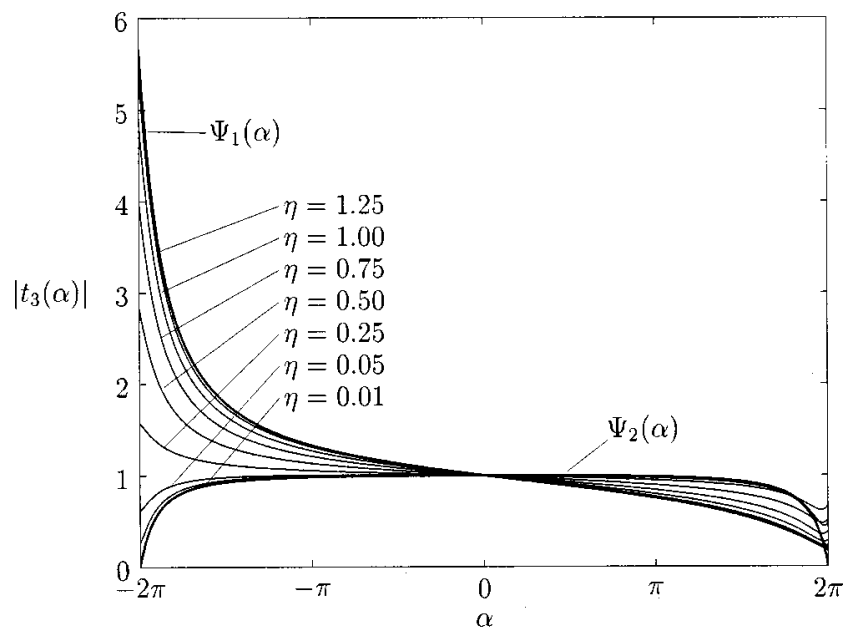

FIG. 10. Magnitude of the branch-free solution $t_{3}(\alpha)$ given in (56) when $\theta=0.25(1+i)$ for various values of $\eta$. The thicker lines corresponds to the known limiting function $\Psi_{1}(\alpha)$, per (11), for $\eta=\pi / 2$ and $\Psi_{2}(\alpha)$, per (13), for $\eta=0$.

\section{CONCLUSION}

A recently proposed solution technique for a class of second order functional difference equations was applied to a case of intermediate complexity in order to assess its potential use for solving certain electromagnetic scattering problems. The essence of this conceptually simple approach lies in the construction of branched solutions to first order difference equations and this is achieved by systematically eliminating singular contributions to produce single-valued expressions. This requirement leads to an equation system whose analytical solution is made possible by obtaining, through the application of Cauchy's theorem on Riemann surfaces, specialized versions of relationships arising in the bilinear relations of Riemann. While the portion of the analysis carried out on a Riemann surface of genus one has the same order of complexity as the one found in Ref. 9, we were also now required to carry out a similar but more intricate analysis on a Riemann surface of genus three in order to obtain well-defined branched solutions to the related first order equations. The final solutions, expressed in terms of branch-free linear combinations of the branched solutions to the first order equations, have all the desired analyticity properties and also recover the known solution $\Psi_{1}(\alpha)$ as $\eta \rightarrow \pi / 2$. The fully analytical approach presented satisfies all of the solution requirements and the fact that it vanishes as $\eta \rightarrow 0$ is not a critical shortcoming since known exact solutions are available in that particular limit. The other variation provided, which requires the numerical identification of zeros in the complex $\alpha$ plane, represents an attempt at resolving this shortcoming and, although it fails when $\eta \sim 0$, it otherwise recovers a solution which varies smoothly between the two known limiting functions $\Psi_{1}(\alpha)$ and $\Psi_{2}(\alpha)$. This is encouraging since it suggests that the proposed approach has the potential to produce a solution that smoothly recovers the two known limiting functions provided a proper method for constructing branch-free solutions can be found.

The results obtained demonstrate the promise of the proposed technique but there are still a large number of interesting issues to be addressed. Indeed, while the procedure for constructing branch-free solutions is fairly well understood, the construction of such meromorphic solutions free of poles and zeros in particular regions of the complex $\alpha$ plane, the strip $\mathcal{S}_{4 \pi}$ in this instance, remains challenging. Consequently, a fully analytical solution displaying the more desirable behavior obtained for the numerical approach, where the solutions recovers $\Psi_{1}(\alpha)$ and $\Psi_{2}(\alpha)$ in the appropriate limits, is still sought. The success of this endeavor is apparently dependent on gaining more insight into the behavior of the zeros of the meromorphic functions constructed. Additionally, a better understanding of the dependency of the quantities $\Lambda_{2 \pi, 4 \pi}$ on the problem parameters $\eta$ and $\theta$ is required. Ideally, this would take the form of specific requirements on, for instance, the 
impedances characterizing the structure and would provide a range over which the procedure for determining the values $\zeta_{2 \pi, 4 \pi}$ can be carried out. Indeed, it is not inconceivable that under certain circumstances $\Lambda_{2 \pi, 4 \pi}$ might lie outside the $\mathcal{P}$ and $\mathcal{Q}$ parallelograms, leading to a failure of the technique. Another highly interesting item is the application of the approach when solutions of different orders (i.e., not $\mathcal{O}(1)$ as $|\operatorname{Im} \alpha| \rightarrow \infty$ ) are required since in such cases the integrand of $w(\alpha, u)$ is required not to vanish as $|\operatorname{Im} \alpha| \rightarrow \infty$. It is, however, apparent that, unless beneficial symmetries can be found in cases of higher complexity, a sufficiently large number of singularities in the strip of analyticity, while not precluding a solution in principle, may well make such an approach impractical. Despite this and some of the currently unresolved issues mentioned above, the technique proposed in Ref. 9, as demonstrated herein, can be applied relatively straightforwardly to cases of intermediate complexity. Current efforts focus on its application to cases of higher complexity such as the diffraction from an anisotropic impedance half-plane illuminated at skew incidence.

\footnotetext{
${ }^{1}$ G. D. Maliuzhinets, Sov. Phys. Dokl. 3, 752 (1958).

${ }^{2}$ A. V. Osipov and A. N. Norris, Wave Motion 29, 313 (1999).

${ }^{3}$ T. B. A. Senior and J. L. Volakis, Approximate Boundary Conditions in Electromagnetics (IEE, London, 1995).

${ }^{4}$ M. Idemen, Wave Motion 32, 37 (2000).

${ }^{5}$ G. D. Maliuzhinets, Sov. Phys. Dokl. 3, 52 (1958).

${ }^{6}$ J. M. L. Bernard, J. Phys. A 31, 595 (1998).

${ }^{7}$ G. Manara and P. Nepa, IEEE Trans. Antennas Propag. 48, 547 (2000).

${ }^{8}$ M. Gaudin, J. Phys. 39, 1143 (1978).

${ }^{9}$ T. B. A. Senior and S. R. Legault, Radio Sci. 35, 683 (2000).

${ }^{10}$ C. Demetrescu, C. C. Constantinou, and M. J. Mehler, Radio Sci. 33, 39 (1998).

${ }^{11}$ G. Springer, Introduction to Riemann Surfaces (Chelsea, New York, 1981).

${ }^{12}$ P. E. Appell, Théorie des Fonctions Algébriques (Chelsea, New York, 1976), Vol. 1.

${ }^{13}$ T. B. A. Senior and S. R. Legault, Electromagnetics 18, 207 (1998).
} 